\title{
Hypersurface normalised gain-scheduled controller for a non-linear 6-DOF fast jet
}

\author{
Jordan Hamilton, David Galvão Wall, Alistair J. Saddington ${ }^{1}$, John T. Economou \\ Aeromechanical Systems Group, Centre for Defence Engineering, \\ Cranfield University, Defence Academy of the UK, Shrivenham, SN6 8LA, UK
}

\begin{abstract}
This paper describes a novel approach for improving the dynamic response of a bank-to-turn autopilot for a non-linear six degree-of-freedom (6-DoF) aircraft model. The autopilot consists of a series of gain-scheduled (GS) proportional, integral and derivative (PID) controllers that govern the aircraft's angular velocities for roll, pitch and yaw. The controller gains have been optimised for localised trim points and applied continuously to the controllers using linear interpolation to form a hypersurface. Our novel solution has been achieved by implementing a set of scheduled gains for near-zero reference signals and integrating this with a set of gains that are normalised to the reference signal. The proposed approach has been compared to conventional gain scheduling techniques using a series of step input simulated manoeuvres, applied individually to the roll and pitch controllers. The results show improved rise and fall times, steady state errors, as well as reduced controller effort.
\end{abstract}

Keywords: hypersurface; gain-scheduling; flight control

\section{Nomenclature}
$b \quad$ wing span $[\mathrm{m}]$
$C_{l, t}, C_{m, t}, C_{n, t}$ aircraft total rolling, pitching and yawing moment coefficients
$C_{x, t}, C_{y, t}, C_{z, t} \quad$ aircraft total force coefficient in the $x, y$ and $z$ directions
$\bar{c} \quad$ wing mean aerodynamic chord $[\mathrm{m}]$
$d A C \quad$ roll rate controller output for aileron command

\footnotetext{
Email address: a.j.saddington@cranfield.ac.uk (Alistair J. Saddington)

${ }^{1}$ Corresponding author.
} 


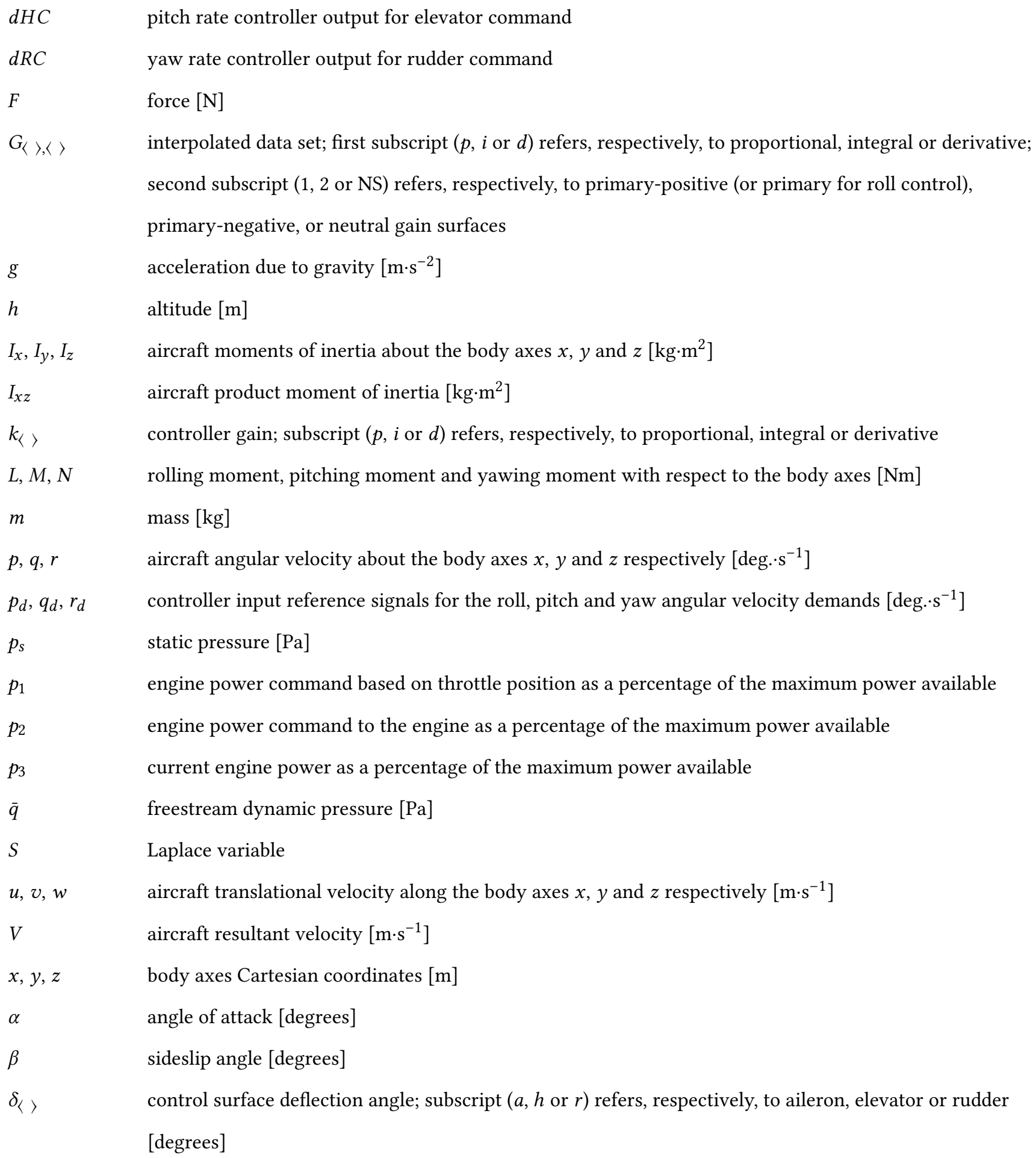




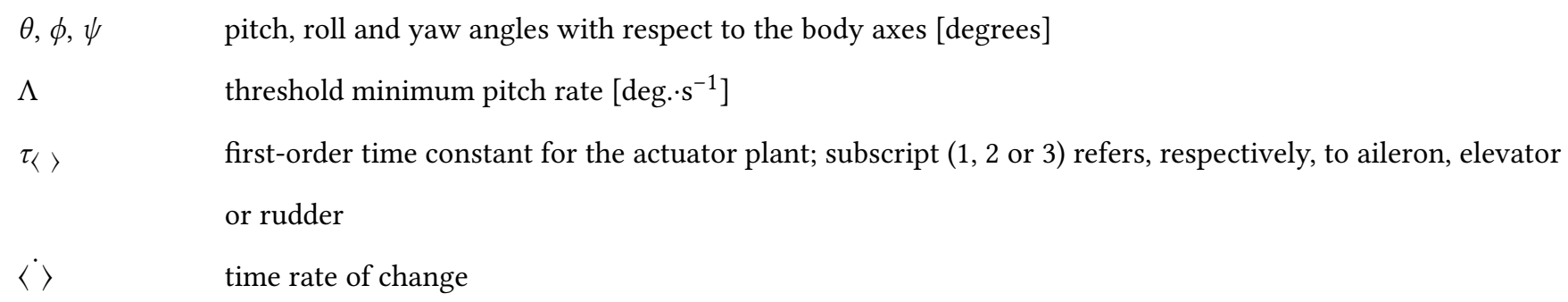

\section{Introduction}

Gain scheduling (GS) is a form of open-loop adaptive control [1] for applications with widely varying non-linear or parameter dependant dynamics [2]. GS has been applied to aircraft autopilots since the end of World War II [1] through the implementation of a series of linear controllers where each linear controller is governed through optimal local control laws for a set of system state dynamics. The performance of an autopilot is highly dependant on the effectiveness of the corresponding control surfaces, which is a function of altitude and Mach number [1]. These two state dynamics can be used to determine a series of local equilibrium trim points. Trim points are defined as a series of system plant dynamic states for which optimisation has been performed to guarantee closed-loop stability, robustness, and ideal dynamic performance characteristics. However, changes that occur to the plant dynamics can cause the state dynamics to transition away from the 
local trim points. This can cause the controller performance to degrade [3] with the potential to induce closed-loop instability. This is fundamentally one of the limitations of GS.

The location and spacing between each trim point is usually estimated by the designer of the system, with the intention of providing closed-loop system stability throughout the entire operating domain. It is advantageous to schedule the gains against slowly-varying dynamic states, such as altitude and airspeed [4], as this will prevent the state dynamics from transitioning too rapidly between the linearised trim points and potentially inducing dynamic instability. However, Richardson et al. [4] argues that it may be desirable to schedule the gains against rapidly-varying states, such as angle of attack. By using angle of attack as a system state and scheduling the gains against it, Richardson et al. [4] created a cyclic dependence. A second-order non-linear longitudinal combat aircraft model with aerodynamic representation was developed using a mathematical smoothing function as opposed to aerodynamic data. Although this will reduce the accuracy and realism of the numerical simulation, the scope of the research was to identify control laws for dynamic gain scheduling (DGS) with state feedback and prove the benefits of scheduling against a system dynamic state rather than a demanded input.

More recently, Yang et al. [5] implemented a DGS controller to a longitudinal dynamic model of the F-16 aircraft. This is a well established and realistic non-linear representation with detailed non-linear aerodynamic data. Yang et al. [5] tested five different controller configurations, each of which scheduled either static or dynamic gains. The state dynamics used for DGS were a mixture of quickly varying states, such as angle of attack, pitch rate or both. Yang et al. [5] concluded that the use of quickly varying dynamic states did not result in large instabilities when scheduling against angle of attack. However, when scheduling solely against pitch rate the controller failed to produce an acceptable response. The response indicated that the controller tended towards instability when the so-called hidden coupling terms within the aerodynamic data (for example those dependent on angle of attack) were not accounted for.

Nichols et al. [6] illustrated an $H_{\infty}$ controller design that would track the commanded normal acceleration inputs of a pitch-axis autopilot for a missile model. The framework for the non-linear controller incorporated rational procedures for scheduling the gains in an effort to reduce the number of linear design trim points whilst still maintaining robustness over the entire operating domain. The controller framework was split into two functions. The first involved computationally parametrising the linear controller by interpolating the poles, zero, and the gains of the trim points. Not only does this linearise the dynamics, allowing equilibrium points to be identified, it also provides the designer with a criterion for the spacing of the operating trim points. The second function calculated a non-linear GS controller that aimed to remove the hidden coupling terms associated with scheduled controllers. These coupling terms prevent the GS system from displaying adequate and 
expected performance between the linearised controller and linearised plant. The removal of the hidden coupling terms was one of the main research focuses, and an analysis was performed against a naive GS controller to show the direct effect of the terms on overall performance. There are limitations with the work conducted by Nichols et al. [6], many of which are highlighted directly by the author. The main limitation towards the application to a generic flight control system is the required linearisation of the model, single state scheduling, and the demand for pole and zero migration in order to configure the controller. The controller did, however, show dynamic improvements to the pitch-rate autopilot, in both the transient and steady-state phase, highlighting the adverse effects of coupling terms in scheduled controllers.

To overcome the stability limitations of GS whilst operating between localised trim points, McConley et al. [7] developed an alternative gain scheduling procedure that would guarantee closed-loop stability throughout the entire operating domain, whilst maintaining some performance characteristics of GS. This was achieved by applying a quadratic robust control Lyapunov function (RCLF) to a target equilibrium trim point, and then computing a region of stability from the RCLF. The same procedure was repeated for any trim points within the operating domain provided that the linearised dynamics about this point could be made stable. Since each trim point defines a region of stability, McConley et al. [7] was able to systematically determine the spacing of each trim point to guarantee closed-loop stability throughout the entire operating domain, with regions of stability "switching" during intersection.

Lu et al. [8] attempted to implement a family of linear parameter varying (LPV) controllers that would enable a fighter aircraft to operate during low or high angle of attack regimes. An aircraft operating in low angle of attack scenarios requires fast and accurate dynamic responses. It is common, however, for fighter aircraft to operate at high angles of attack in near-stall or post-stall regimes. This requires a different operating controller where the emphasis lies on maintaining stability and acceptable flying qualities. It is very difficult to develop a single LPV controller that can operate over this range of flight dynamics. Lu et al. [8] proposed, therefore, a family of LPV controllers that switch according to the evolution of angle of attack. Each of the LPV controllers achieved operating stability regions through the use of Lyapunov functions, however, the use of multiple Lyapunov functions, coupled with controller switching meant that whenever a switch occurred the state controller had to be reset to guarantee stability within the next operating region. This was not always a feasible solution as a lack of plant state information would result in the controller having to perform an operating estimation by adding an observer. Furthermore, to avoid possible transient instabilities associated with LPV discontinuous switching, Lu et al. [8] initiated the switching using two parameter-dependant switching logics: hysteresis switching; and switching with an average dwell-time. 
Tran et al. [9] integrated a series of linear gain scheduled controllers into a non-linear longitudinal F-18 High Alpha Research Vehicle (HARV) model. Although the dynamics of the HARV model were linearised using small angle perturbations, the aerodynamics were highly non-linear. Tran et al. [9] developed two controller scenarios: a linear control augmentation system (CAS) controller and a linear/non-linear stabilisation augmentation system (SAS) controller. The CAS design utilised a proportional-integral (PI) controller architecture to feedback attitude angle errors. The SAS design was split into two variants. The first variant was a linear SAS designed to feedback angle of attack and pitch rate dynamics. The latter was a non-linear SAS designed to deal with nonlinearities due to changes within the aerodynamics. Both CAS and SAS designs were gain-scheduled about a series of linearised trim points.

One of the main issues with conventional GS is the switching of gains that occurs at the boundaries of neighbouring trim points. It has been discussed how the use of slowly-varying dynamic states can alleviate the effects of gain switching, as well as how systematically determining the trim point spacing can prevent dynamic instability from occurring during this transition. In each case, however, the transition is highly discontinuous and this can cause large fluctuations in the controller output. Stilwell and Rugh [10] developed an interpolation method for a linear gain-scheduled controller that would enable a continuous gain change between neighbouring trim points. A schematic showing GS and a linearly-interpolated gain surface can be seen in Figure 1. A similar approach was implemented by Galvão Wall [11] to tune a series of linear controllers for a non-linear three degree-of-freedom robotic manipulator. Theodoulis et al. [12] implemented GS into a $H_{\infty}$ pitch/yaw channel autopilot for a $155 \mathrm{~mm}$ spin-stabilised guided projectile. To prevent discontinuities, smooth out the gain profiles, and generate a reasonable amplitude, the controller gains were interpolated online using lookup tables. Furthermore Choi et al. [13] developed a polynomial regression model to replace the nearest neighbour and interpolation algorithms for intermediate state dynamics. Choi et al. [13] hypothesised that generating a polynomial regression would reduce the computational costs associated with bilinear interpolation, and the controller degradation associated with the nearest neighbour algorithm.

Control law interpolation was also used by McConley et al. [7] to develop a continuous state trajectory throughout individual regions of stability. However, interpolation was not performed between each individual control law, resulting in discontinuous gain switching for these small regions of operation. Wu and Kim [14] implemented controller interpolation for an LPV GS controller with the aim of improving performance over the operating domain. This methodology involved the use of localised Lyapunov functions developed for operating trim points with interpolation performed against a global Lyapunov function to generate a continuous gain profile throughout the operating domain. Additionally, research was conducted into reducing the implications on stability whilst transitioning across neighbouring gain sets. Apkarian and Adams [15] have 


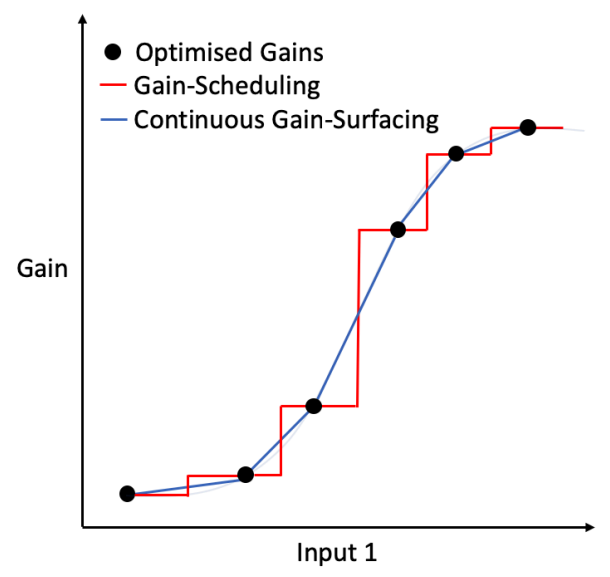

Figure 1: Comparison of gain scheduling and continuous gain-scheduling schematic.

implemented a linear matrix inequalities (LMI) and a Lyapunov function to a series of controller gains to allow a smoother transition between the various sets, with the aim of improving stability. This method was deemed conservative by Shirnomura [16] as it reduces the controller's rate of change, effectively throttling the response to prevent a non-smooth transition from negatively impacting the system.

Whilst GS remains one of the most popular control techniques for non-linear systems, it has been discussed how the limitations of GS, primarily due to gain switching, can cause closed-loop instability. GS can also negatively impact dynamic performance when operation occurs outside of the linearised trim points. Although the problems associated with GS can be somewhat alleviated using an interpolation approach to generate a continuous gain-surface (CGS), the dynamic performance in some cases is not improved. In this paper we present a novel approach for determining the real-time controller gains for dynamic-state feedback using a proportional-integral-derivative (PID) controller. This technique is applied to a six degree of freedom (6-DoF) non-linear aircraft model that is representative of a fighter jet. The PID controllers applied to the model control the aircraft's angular rates of change for pitch, roll and yaw.

The paper is organised as follows. In Section 2 the aircraft dynamic model is introduced. This section describes the non-linear equations of motion, aerodynamics, and kinematic control surface models. The control system architecture is also introduced as well as the approach to the optimisation of the controller gains and the location of the trim points. Section 3 outlines the computational simulations that were performed on not only the two methods discussed during the introduction (traditional GS and CGS) but also on our improved method. Since GS and CGS are already well established techniques, both methodologies were validated against the existing literature, with the results presented in Appendix A. In Section 4 each 
stage of improvement over the established techniques is introduced and the mathematical logic is explained. The results and discussion surrounding our approach are presented in Section 5.

\section{Dynamic model}

In this section we introduce the control architecture, the non-linear 6-DoF aircraft model, optimisation method and selection of trim points. Figure 2 shows a condensed block diagram for the overall structure of the model. There are four main areas shown within the block diagram: non-linear equations of motion; non-linear aerodynamics; aircraft control system; and the gain-scheduling block. The research novelty focuses on the highlighted gain-scheduling block and the interface between the scheduler and the controllers. The control techniques discussed in Section 1, namely, dynamic-state gain-scheduling and continuous gain-scheduling, were applied to the model to generate a benchmark controller performance. This benchmark is used to demonstrate the benefits of the novel approach.

\subsection{6-DoF aircraft model}

The aircraft is a 6-DoF dynamic model. Each of the six degrees-of-freedom have no association with elasticity, i.e. there is no fuselage bending or wing flexure due to static or dynamic loading. As a result, three translational and three rotational equations of motion (Equation 1) can be derived with a body-axis coordinate system (Figure 3) coincident with the aircraft's centre of gravity (CG). The velocity analysis conducted for rotational motion characterises the roll rate $(p)$, pitch rate $(q)$ and yaw rate $(r)$ of the aircraft with respect to the CG. The equations of motion are obtained using Newton's laws with vector differentiation [17]. Each equation of motion (Equation 1) has a force $\left(F_{x}, F_{y}, F_{z}\right)$ or moment $(L, M, N)$ that can be broken down into individual components that represent the aerodynamic forces and thrust forces acting in the body axis.

$$
\begin{array}{lll}
\dot{u}=r v-q w-g \sin \theta+\frac{F_{x}}{m} ; & \dot{v}=p w-r u-g \sin \phi \cos \theta+\frac{F_{y}}{m} ; & \dot{w}=q u-p v-g \cos \phi \cos \theta+\frac{F_{z}}{m} \\
\dot{p}=\left(c_{1} r+c_{2} p\right) q+c_{3} L+c_{4} N ; & \dot{q}=c_{5} p r-c_{6}\left(p^{2}-r^{2}\right)+c_{7} M ; & \dot{r}=\left(c_{8} p-c_{2} r\right) q+c_{4} L+c_{9} N
\end{array}
$$

The moments of inertia, $I_{x}, I_{y}$ and $I_{z}$ and the product moment of inertia, $I_{x z}$ are calculated with respect to the aircraft's body-axis. The aircraft is assumed to be symmetrical about the $x-z$ axis and, therefore, the product moments of inertia $I_{x y}$ and $I_{y z}$ are zero. The terms relating to the moments of inertia are presented in Equation 2. 


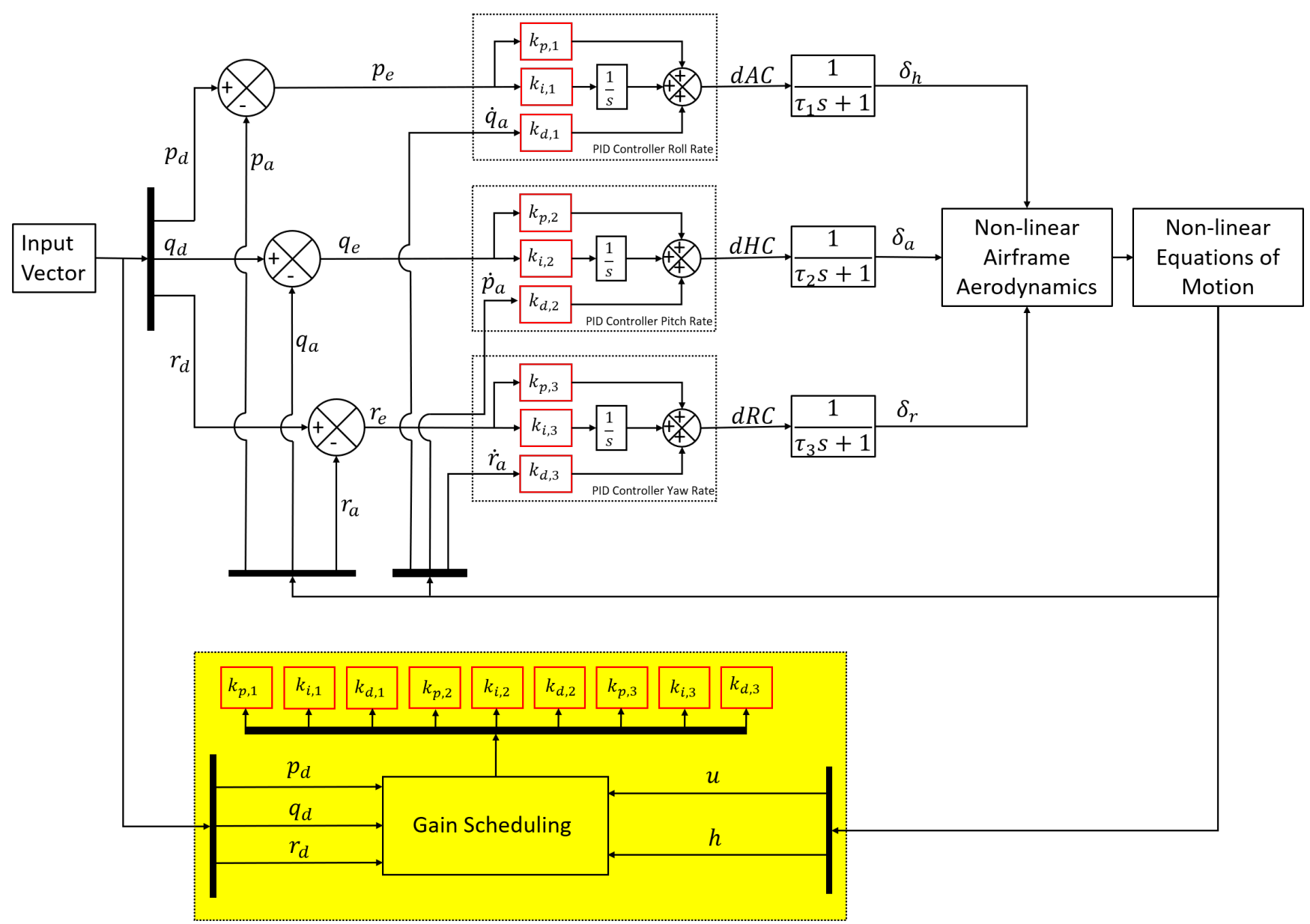

Figure 2: Aircraft control system block diagram. Signals $\delta_{h}, \delta_{a}$ and $\delta_{r}$ are saturated to reflect control deflection limits. These limits are described in Table 1 .

$$
\left.\begin{array}{rlrl}
c_{1}=\frac{\left(I_{y}-I_{z}\right) I_{z}-I_{x z}{ }^{2}}{I_{x} I_{z}-I_{x z}{ }^{2}} ; & c_{4}=\frac{I_{x z}}{I_{x} I_{z}-I_{x z}{ }^{2}} ; & c_{7}=\frac{1}{I_{y}} \\
c_{2}=\frac{\left(I_{x}-I_{y}+I_{z}\right) I_{x z}}{I_{x} I_{z}-I_{x z}{ }^{2}} ; & c_{5}=\frac{I_{z}-I_{z}}{I_{y}} ; & c_{8}=\frac{I_{x}\left(I_{x}-I_{y}\right)+I_{x z}{ }^{2}}{I_{x} I_{z}-I_{x z}{ }^{2}} \\
c_{3}=\frac{I_{z}}{I_{x} I_{z}-I_{x z}{ }^{2}} ; & c_{6}=\frac{I_{x z}}{I_{y}} ; & c_{9}=\frac{I_{x}}{I_{x} I_{z}-I_{x z}{ }^{2}}
\end{array}\right\}
$$

Nguyen et al. [19] published a series of 50 subsonic multidimensional lookup tables. These were populated using wind tunnel test data on a scale model of an F-16 aircraft and arranged based on surface deflection, angle of attack, and sideslip 


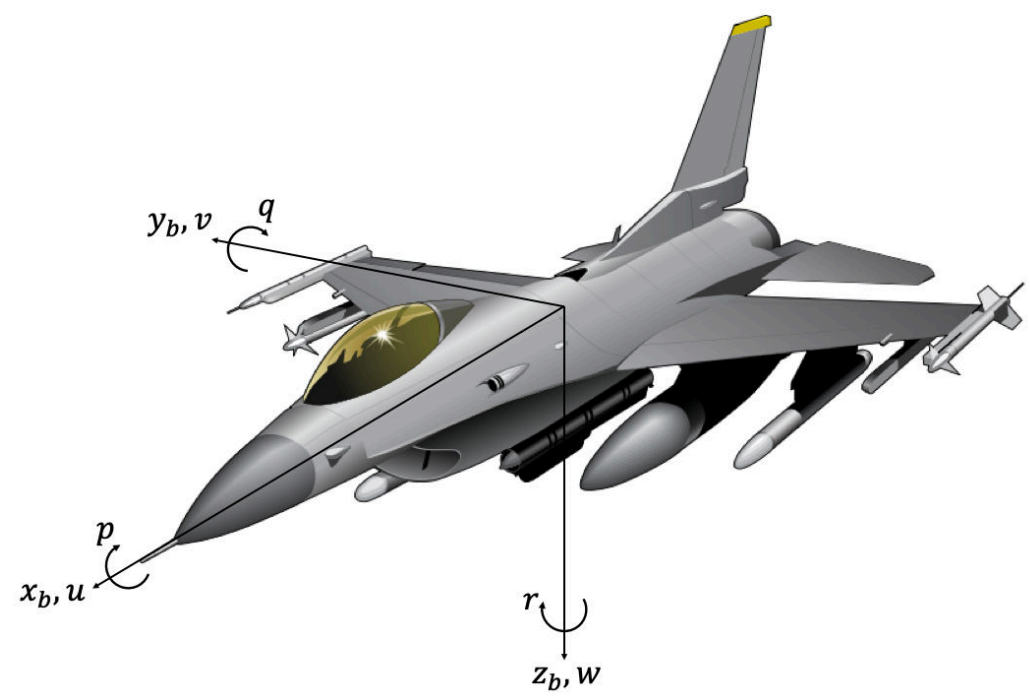

Figure 3: Aircraft model axis system (adapted from Wikimedia Commons [18]).

angle. The data covers a range of angles of attack from $-20^{\circ}$ to $90^{\circ}$ and sideslip angles from $-30^{\circ}$ to $30^{\circ}$. Researchers, such as $\mathrm{Lu}$ [20], Savran et al. [21] and Omran et al. [22] state that this operating window is beyond the scope of many dynamic modelling problems. As a result they have used data published by Stevens et al. [17]. This aerodynamic dataset is described by $\mathrm{Lu}[20]$ as "approximated data" which was derived from only ten of the aerodynamic lookup tables published by Nguyen et al. [19]. The aerodynamic coefficients derived from the approximated data do not include the effect of the leading-edge flaps, and reduce the operating angle of attack range to $-10^{\circ}$ to $45^{\circ}$. Furthermore, the use of these lookup tables requires online linear interpolation to obtain the aerodynamic data during intermediate state dynamics. Work by Morelli [23] aimed to eliminate the need to perform online linear interpolation by using multivariate orthogonal modelling functions. This parametric modelling technique was applied to the wind tunnel data of Nguyen et al. [19] to generate a non-linear model structure, which expands into an ordinary multivariate polynomial. Whilst largely successful, there was up to a $10 \%$ difference between the aforementioned approximated wind tunnel test data and the non-linear parametrised model whilst performing a simulated doublet manoeuvre [23]. In our 6-DoF model we have not made these assumptions and have incorporated the entire non-linear multidimensional dataset published by Nguyen et al. [19] with online linear interpolation. Hence, the six non-linear subsonic aerodynamic coefficients $\left(C_{x, t}, C_{y, t}, C_{z, t}, C_{l, t}, C_{m, t}\right.$ and $\left.C_{n, t}\right)$ are determined from Equations 3 to 8. Each of the terms in the equations is a function of a series of datasets that have been normalised against surface deflections. 


$$
C_{x, t}=C_{x}\left(\alpha, \beta, \delta_{h}\right)+\Delta C_{x, \text { lef }}\left(1-\frac{\delta_{\text {lef }}}{25}\right)+\Delta C_{x, s b}(\alpha)\left(\frac{\delta_{s b}}{60}\right)+\frac{\bar{c} q}{2 V}\left[C_{x_{q}}(\alpha)+\Delta C_{x_{q, \text { lef }}}(\alpha)\left(1-\frac{\delta_{\text {lef }}}{25}\right)\right]
$$

$$
\begin{aligned}
C_{y, t}=C_{y}(\alpha, \beta)+\Delta C_{y, \text { lef }}\left(1-\frac{\delta_{\text {lef }}}{25}\right)+\left[\Delta C_{y, \delta_{a=20}}+\Delta C_{y, \delta_{a=20}, \text { lef }}\left(1-\frac{\delta_{\text {lef }}}{25}\right)\right]\left(\frac{\delta_{a}}{20}\right)+ \\
\Delta C_{y, \delta_{r=30}}\left(\frac{\delta_{r}}{30}\right)+\frac{b}{2 V}\left\{\left[C_{y_{r, \text { lef }}}(\alpha)\left(1-\frac{\delta_{\text {lef }}}{25}\right)\right] r+\left[C_{y_{p}}(\alpha) \Delta C_{y_{p, \text { lef }}}(\alpha)\left(1-\frac{\delta_{\text {lef }}}{25}\right)\right] p\right\}
\end{aligned}
$$

$C_{z, t}=C_{z}\left(\alpha, \beta, \delta_{h}\right)+\Delta C_{z, \text { lef }}\left(1-\frac{\delta_{\text {lef }}}{25}\right)+\Delta C_{z, s b}(\alpha)\left(\frac{\delta_{s b}}{60}\right)+\frac{\bar{c} q}{2 V}\left[C_{z_{q}}(\alpha)+\Delta C_{z_{q, \text { lef }}}(\alpha)\left(1-\frac{\delta_{\text {lef }}}{25}\right)\right]$

$$
\begin{gathered}
C_{l, t}=C_{l}\left(\alpha, \beta, \delta_{h}\right)+\Delta C_{l, \text { lef }}\left(1-\frac{\delta_{\text {lef }}}{25}\right)+\left[\Delta C_{l, \delta_{a=20}}+\Delta C_{l, \delta_{a=20}, \text { lef }}\left(1-\frac{\delta_{\text {lef }}}{25}\right)\right]\left(\frac{\delta_{a}}{20}\right)+ \\
\Delta C_{l, \delta_{r=30}}\left(\frac{\delta_{r}}{30}\right)+\frac{b}{2 V}\left\{\left[C_{l_{r, \text { lef }}}(\alpha)\left(1-\frac{\delta_{\text {lef }}}{25}\right)\right] r+\left[C_{l_{p}}(\alpha) \Delta C_{l_{p, \text { lef }}}(\alpha)\left(1-\frac{\delta_{\text {lef }}}{25}\right)\right] p\right\}+\Delta C_{l_{b}}(\alpha) \beta
\end{gathered}
$$

$$
\begin{gathered}
C_{m, t}=C_{m}\left(\alpha, \beta, \delta_{h}\right) \eta_{\delta_{h}}\left(\delta_{h}\right)+C_{z, t}\left(x_{\mathrm{CG}, r e f}-x_{\mathrm{CG}}\right)+\Delta C_{m, \text { lef }}\left(1-\frac{\delta_{\text {lef }}}{25}\right)+ \\
\Delta C_{m, s b}(\alpha)\left(\frac{s b}{60}\right)+\frac{\bar{c} q}{2 V}\left[C_{m_{q}}(\alpha)+\Delta C_{m_{q, \text { lef }}}(\alpha)\left(1-\frac{\delta_{\text {lef }}}{25}\right)\right]+\Delta C_{m}(\alpha)+\Delta C_{m, d s}\left(\alpha, \delta_{h}\right)
\end{gathered}
$$

$$
\begin{aligned}
C_{n, t}=C_{m}\left(\alpha, \beta, \delta_{h}\right)+\Delta C_{n, \text { lef }}\left(1-\frac{\delta_{\text {lef }}}{25}\right)+\left[\Delta C_{n, \delta_{a=20}}+\Delta C_{n, \delta_{a=20, \text { lef }}}\left(1-\frac{\delta_{\text {lef }}}{25}\right)\right]\left(\frac{\delta_{a}}{20}\right)+ \\
\Delta C_{n, \delta_{r=30}}\left(\frac{\delta_{r}}{30}\right)+\frac{b}{2 V}\left\{\left[C_{n_{r, \text { lef }}}(\alpha)\left(1-\frac{\delta_{\text {lef }}}{25}\right)\right] r+\left[C_{n_{p}}(\alpha) \Delta C_{n_{p, \text { lef }}}(\alpha)\left(1-\frac{\delta_{\text {lef }}}{25}\right)\right] p\right\}
\end{aligned}
$$


The non-linear kinematics given in Equation 9 are used to generate the correct aircraft orientation when performing vector transformations from the body-axis to the inertial frame [17].

$$
\left.\begin{array}{l}
\dot{\phi}=p+\tan \theta(q \sin \phi+r \cos \phi) \\
\dot{\theta}=q \cos \phi-r \sin \theta \\
\dot{\psi}=\frac{q \sin \phi+r \cos \phi}{\cos \theta}
\end{array}\right\}
$$

The aircraft engine has been modelled with first-order dynamics, however, the time constant is variable depending on the difference between the actual power and the power demand. Figure 4 shows a logic tree depicting the dynamics of the first-order engine model. This data is taken from Nguyen et al. [19] and is the same engine model used by Stevens et al. [17] in their linearised F-16 model and by Albostan and Göka [24] in their decoupled lateral-directional analysis of the F-16.

An airspeed controller has been incorporated into the model that regulates the throttle to maintain, increase, or decrease the airspeed of the aircraft. The airspeed controller has the same PID architecture as the other controllers and was optimised through the same process, however, it has a variable integral component that engages and disengages depending on the current flight parameters and the position error within the system. This functionality was vital as the engine has relatively slow dynamics due to the variable first-order lag within the model. As a consequence, as the input demand changes, integral wind-up occurs resulting in large errors accumulating over time.

The aircraft model has four sets of control surfaces: ailerons; elevators; rudder; and leading-edge flaps. The three primary control surfaces: ailerons; elevators; and rudder are regulated individually using PID controllers. The leading-edge flaps are scheduled against angle of attack and the static and dynamic pressures, as denoted by Equation 10 [19].

$$
\delta_{\text {lef }}=1.38 \frac{2 S+7.25}{S+7.25} \alpha-9.05 \frac{\bar{q}}{p_{s}}+1.45
$$

The PID controllers use negative feedback to control the aircraft angular velocities about the roll, pitch and yaw axes. The aircraft angular acceleration is used as the derivative error for the PID controller and is obtained numerically from the equations of motion. The output from each of the PID controllers is the input to the corresponding surface actuator plant. Each actuator plant was modelled as a first-order lag with rate and deflection limits. The surface deflections were computed 


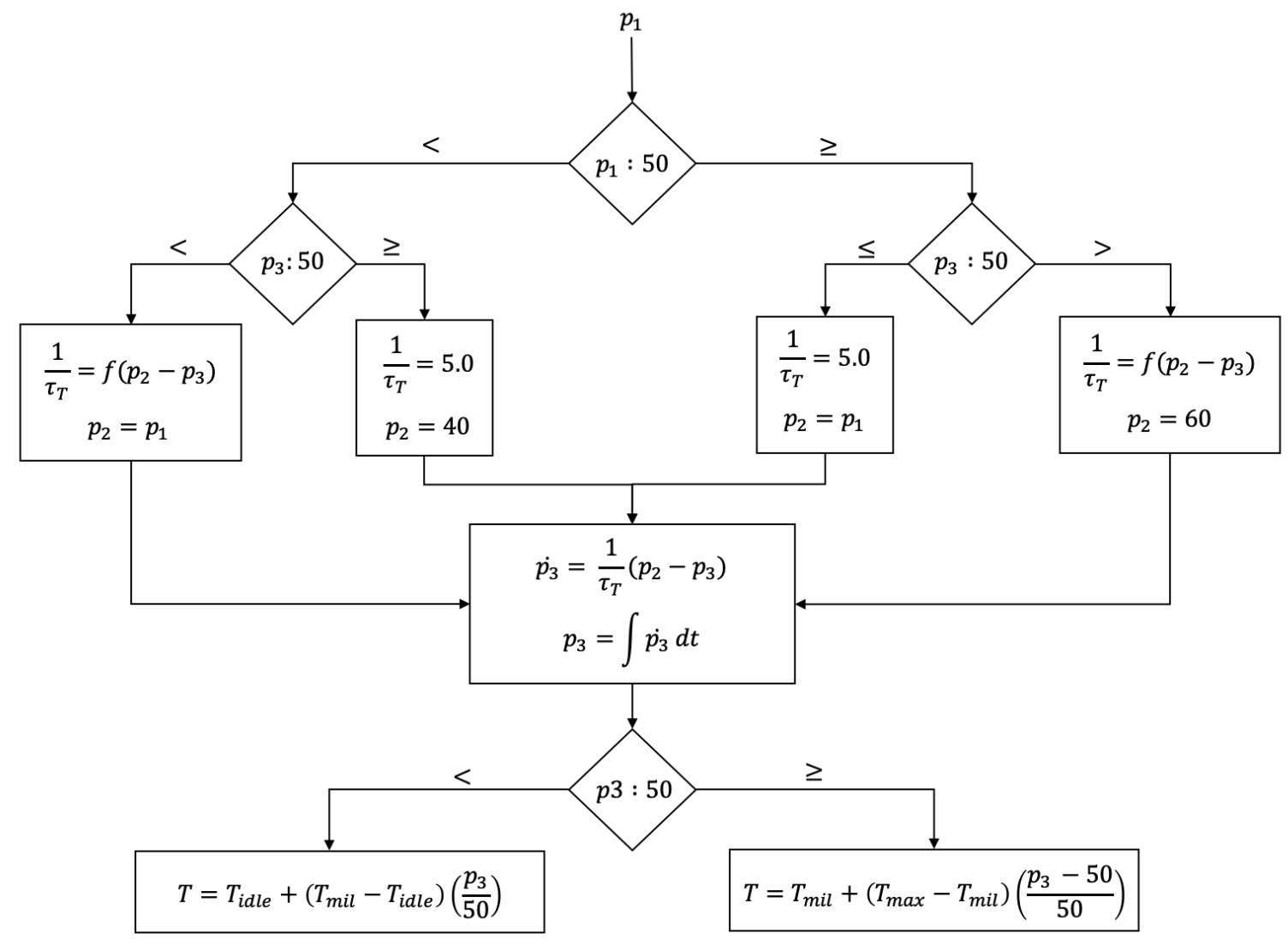

Figure 4: First-order engine model logic tree based on Nguyen et al. [19].

numerically using a Runge-Kutta fourth-order integration [17], and limited both in position and rate of change in accordance with the data from Nguyen et al. [19]. The control surface deflection and rate limits, as well as the first-order time constant, are shown in Table 1.

\begin{tabular}{lccc}
\hline Control Surface & Time Constant $(\mathrm{s})$ & Rate Limit $(\mathrm{deg} / \mathrm{s})$ & Deflection Limit $(\mathrm{deg})$ \\
\hline Aileron & 0.0495 & 80 & \pm 25 \\
Elevator & 0.0495 & 120 & \pm 30 \\
Rudder & 0.1360 & 25 & \pm 25 \\
\hline
\end{tabular}

Table 1: Control surface time constants, rate limits and deflection limits. These limits are implemented as signal saturations on $\delta_{h}, \delta_{a}$ and $\delta_{r}$ in Figure 2. 


\subsection{Controller Optimisation}

Optimisation of the PID controllers was performed using the bat algorithm (BA) to determine the proportional, $K_{p}$, integral, $K_{i}$, and derivative, $K_{d}$, gains for the 24 localised linear trim points shown in Figure 5 . The BA is a meta-heuristic algorithm developed by Yang [25] and combines the dynamics of particle swarm and harmony search algorithms. The use of the BA for optimisation in the current study was the result of an in-depth analysis. Firstly, the topology of the search space contained numerous regions of local minima, causing optimisation algorithms such as gradient descent to struggle locating the global minimum. When comparing a genetic algorithm and BA using a set of optimisation functions, the genetic algorithm showed $99 \%$ accuracy compared with $92 \%$ accuracy for the BA when trying to locate the global minimum. However, the computational time required for the genetic algorithm (GA) to converge was almost 60 times larger than for the BA, which agrees with similar findings of Yang [25]. We decided, therefore, that the $7 \%$ improvement in accuracy was outweighed by the fact that the BA could perform up to 60 optimisations within the same time period as the GA, allowing for a higher resolution optimisation to be achieved.

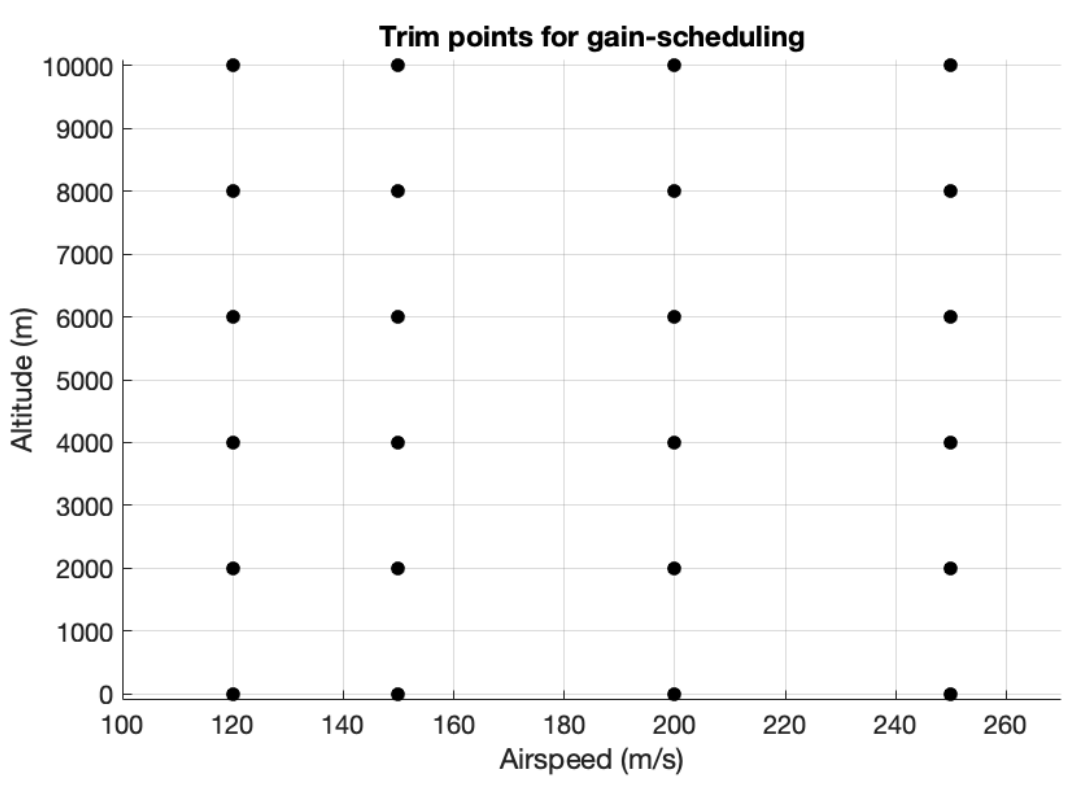

Figure 5: The gain-scheduled trim points used for optimisation.

The location of the trim points was determined by initially optimising the aircraft model for the lowest demanded airspeed $\left(120 \mathrm{~m} \cdot \mathrm{s}^{-1}\right)$ and altitude (0 metres). To optimise the PID controller gains for each trim point the dynamic response of the system to a step input was compared to a designed dynamic response for the same input. For each trim point the amplitude of 
the step input was selected as the maximum possible angular rate at the airspeed and altitude corresponding to that particular trim point. The fitness function applied to the optimisation was a weighted sum-squared error (WSSE) between the step response of the tuned system and the equivalent designed response. Starting with the first trim point at the lowest airspeed and altitude, the optimised gains were maintained while the altitude was increased until the performance of the controller degraded. This altitude was rounded to the nearest $100 \mathrm{~m}$ and selected as the next altitude trim point where the gains were re-optimised. The same procedure was carried out for airspeed rounded to the nearest $10 \mathrm{~m} \cdot \mathrm{s}^{-1}$. Although the trim points were not systematically determined to guarantee stability when using GS, they were identified through the optimisation process as being within close enough proximity to not induce dynamic instability whilst operating with intermediate state dynamics.

The roll rate and yaw rate controllers were both optimised for a single set of gains, due to symmetry about the operating axes. This gain set will be referred to as the primary gain set, however, the pitch rate controller required two primary gain sets; one for negative pitch rate demands and one for positive pitch rate demands. For reasons that will become clear later in this paper these will be called the primary-positive and primary-negative gain sets. This multi-level gain scheduling was achieved through an additional layer of logical operations that switches the operating gain set dependant on the input vector.

Each of the rate controllers can be controlled via either a manual piloted stick input, or through a demand instructed by the flight computer. The flight computer is made up of a series of position autopilots which wrap around each of the rate controllers, governing the angular position. The autopilots that control the aircraft angular position can be engaged or disengaged at any moment, allowing a change between autonomous flight and manual flight. This permitted an altitude controller to govern the pitch angle of the aircraft, allowing either an altitude hold, or a change in altitude to commence autonomously. When engaged, these autopilots operate as position controllers that provide reference signals to the rate controllers, which in turn deflect the control surfaces. The autopilots are required for future research purposes using the aircraft dynamic model. For the purpose of this research, we only engaged the altitude controller during the initial stages of the simulation to achieve straight-and-level-flight prior to performing a controlled manoeuvre.

\section{Simulation procedure}

To develop the benchmark against which the performance of the novel approach was tested, a series of simulations was conducted for each angular rate controller individually. All three controllers (pitch rate, roll rate and yaw rate) remain active throughout all manoeuvres. When the simulation started, a series of lookup tables were initialised that give the aircraft 
trimmed conditions. For simulations testing the roll rate controller, the aircraft model was initialised with an airspeed of $175 \mathrm{~m} \cdot \mathrm{s}^{-1}$ and an altitude of 5000 metres. These dynamics were chosen as they are offset from any optimised trim points, enabling an assessment of the performance of a gain set away from its designed trim point. For simulations testing the pitch rate controller, the aircraft model was initialised with an airspeed of $200 \mathrm{~m} \cdot \mathrm{s}^{-1}$ and an altitude of 5000 metres. This placed the aircraft model with flight conditions at an optimised trim point for airspeed and between two trim points for altitude. The increase in airspeed was required for testing the gain scheduler on the pitch rate controller because it involves multiple positive and negative input demands, causing the airspeed to reduce over the course of the manoeuvre. By starting the simulation with a higher airspeed, the aircraft flight dynamics varied sufficiently as the airspeed bled off to transition between optimised trim points. At the start of each benchmark test the airspeed and altitude controllers were enabled to maintain wings-level flight for the given starting conditions. These controllers were then disengaged prior to the manoeuvre commencing. This process eliminates any contrasting flight data that could occur during dynamic initialisation.

Three roll rate manoeuvres were conducted, each of which focused on the aircraft's ability to perform a $360^{\circ}$ roll at a series of constant angular velocities. Each of the three manoeuvres, depicted in Figure 6, start from the same flight conditions; the only differences being the input demand. The first roll manoeuvre (Figure 6(a)) is a $60 \mathrm{deg} / \mathrm{s} \mathrm{roll} \mathrm{for} 6 \mathrm{~s}$. This tests the steady-state error in the controller and its ability to maintain a constant angular velocity for a period of time. The second roll manoeuvre (Figure 6(b)) tests the controller's speed and ability to maintain a constant velocity. The final roll manoeuvre (Figure 6(c)) tests the aircraft's stability and speed as the controller demands a roll rate of $180 \mathrm{deg} / \mathrm{s}$ for only two seconds.

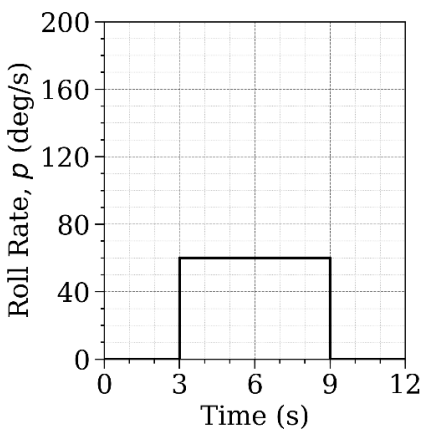

(a) Manoeuvre 1: $60 \mathrm{deg} / \mathrm{s}$ for $6 \mathrm{~s}$.

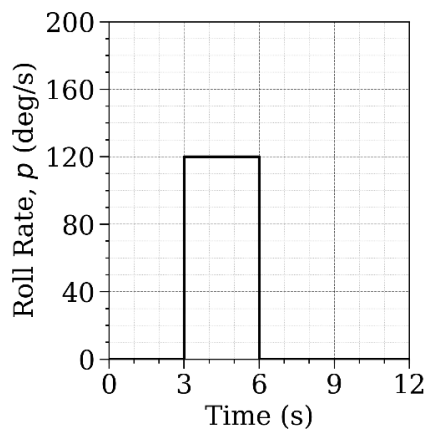

(b) Manoeuvre 2: $120 \mathrm{deg} / \mathrm{s}$ for $3 \mathrm{~s}$.

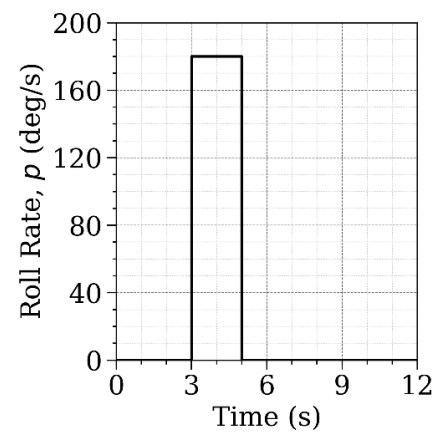

(c) Manoeuvre 3: $180 \mathrm{deg} / \mathrm{s}$ for $2 \mathrm{~s}$.

Figure 6: The three manoeuvres used to assess the aircraft's roll-rate controller performance.

Figure 7 shows the pitch rate manoeuvre, which focuses less on the speed of the controller and more on the controller's robustness when encountering rapidly-changing state dynamics. As the aircraft pitches up the aerodynamic forces acting 
on the aircraft change. This causes the airspeed and altitude (the two state dynamics that the gains are scheduled against) to also change. To prevent the aircraft from stalling during the manoeuvre the throttle was increased to maximum as the airspeed controller was disengaged. This allowed the airspeed of the aircraft to move through various trim points as the aircraft accelerates, inducing a change in gains.

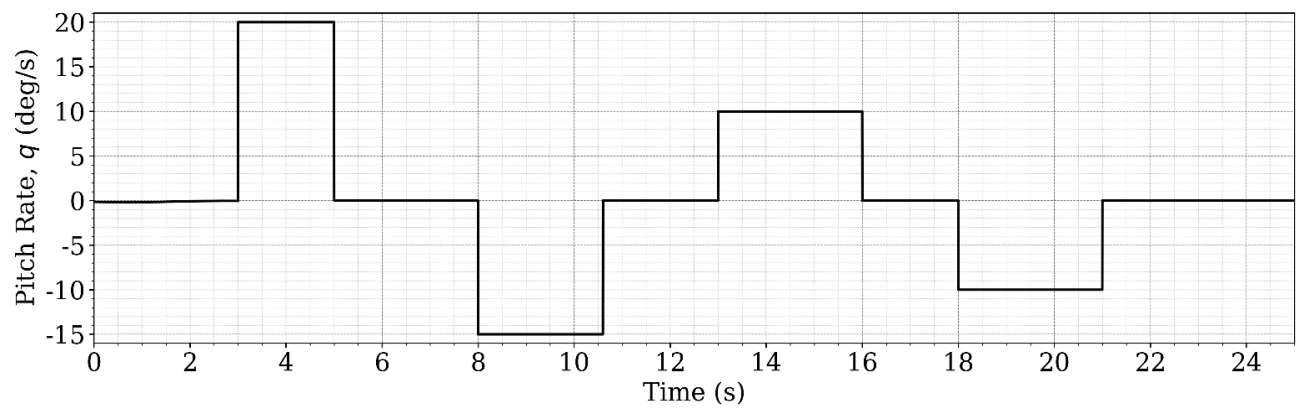

Figure 7: The pitch rate manoeuvre used to assess the aircraft's response to changing dynamic states.

A discussion of the benchmark results is provided in Appendix A. These are now used as a comparison to our proposed improvement to the dynamic response of the system.

\section{Proposed approach}

We now present our approach to improve the dynamic response of the system. It was explained in Section 2.2 that one set of gains was obtained for the roll rate controller and two sets of gains were obtained for the pitch rate controller (positive and negative pitch rate). The gains were optimised using a single step input where the amplitude of the input was the maximum attainable angular rate given by the aircraft flight conditions.

\subsection{Continuous multi-gain-surfaces}

To improve the transient response of the aircraft roll and pitch dynamics when the input reference signal was zero, or approaching zero, a secondary gain surface was implemented that only engages when the magnitude of the reference signal falls below a threshold value. The first approach we present, continuous multi-gain-surfaces (CMGS), uses this approach and aims to generate a multi-surface configuration using primary and secondary surfaces, allowing the controller gains to be scheduled against the reference signal as well as the flight conditions (Figure 8). Furthermore, this is intended to reduce the controller effort as the logical operator assigned to the controller switches to a more appropriate gain surface during intervals of low demand or no demand inputs, whilst maintaining an identical or improved rise-time and steady-state response. 


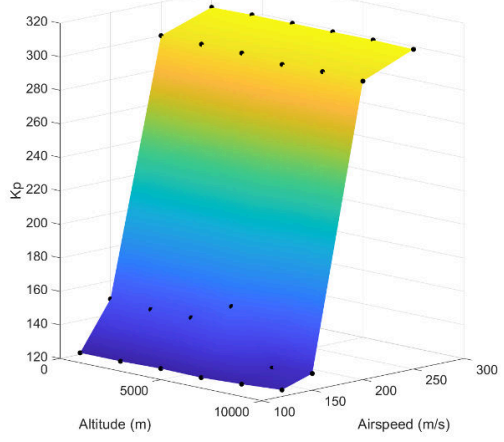

(a) Primary-positive gain surface.

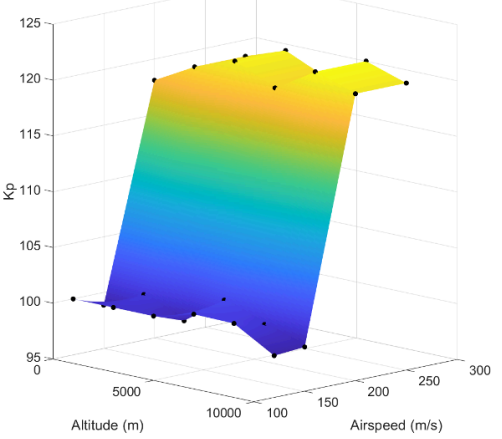

(b) Primary-negative gain surface.

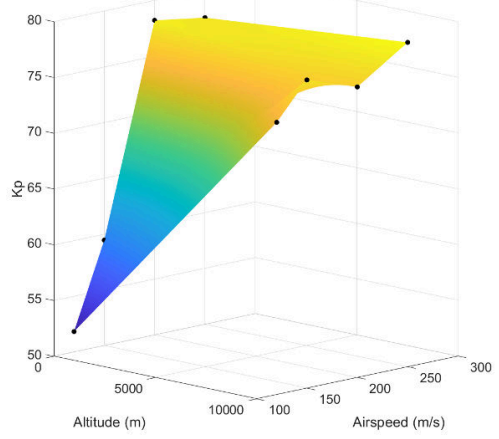

(c) Secondary (neutral) gain surface.

Figure 8: Proportional gain surfaces for the CMGS pitch rate controller.

Although CMGS appears to induce large instantaneous gain changes, contradicting the improvements made through CGS, the controller changes do not occur during a manoeuvre and are only activated when the input vector is equal to zero or is approaching zero. As a result this does not affect the gains or the dynamic response during a manoeuvre. This method increases dynamic performance of the aircraft by reducing the fall time of its post-manoeuvre response when returning its angular velocity to zero, as well as reducing the controller effort in the process. The same bilinear interpolation performed in CGS is active across all gain surfaces, maintaining a continuous gain profile whilst a manoeuvre is performed.

The logic equations for CMGS describe the input vector and switch gain sets dependant on the magnitude of the reference signal. The roll rate controller only requires two components whilst the pitch rate controller requires three as it has positive and negative pitch rate gain surfaces. The roll rate controller has, therefore, a single primary gain surface and a neutral gain surface. For the rolling moment,

$$
\left[\begin{array}{l}
k_{p} \\
k_{i} \\
k_{d}
\end{array}\right]=\left[\begin{array}{l}
G_{p, 1} \cdot\left(\left|p_{d}\right|>0\right)+G_{p, N S} \cdot\left(p_{d}==0\right) \\
G_{i, 1} \cdot\left(\left|p_{d}\right|>0\right)+G_{i, N S} \cdot\left(p_{d}==0\right) \\
G_{d, 1} \cdot\left(\left|p_{d}\right|>0\right)+G_{d, N S} \cdot\left(p_{d}==0\right)
\end{array}\right]
$$

where $G$ represents the bilinear interpolated value from an optimised gain set. The first subscript of $G$ describes the corresponding controller component $(p, i, d)$. The second subscript of $G$ describes whether the gain surface is representing the primary surface or the neutral surface, $(1, N S)$. For example, $G_{p, 1}$ would be the bilinear interpolated value for the primary 
proportional gain set. Finally, for the pitching moment,

$$
\left[\begin{array}{l}
k_{p} \\
k_{i} \\
k_{d}
\end{array}\right]=\left[\begin{array}{l}
G_{p, 1} \cdot\left(q_{d}>0\right)+G_{p, 2} \cdot\left(q_{d}<0\right)+G_{p, N S} \cdot\left(q_{d}==0\right) \\
G_{p, 1} \cdot\left(q_{d}>0\right)+G_{p, 2} \cdot\left(q_{d}<0\right)+G_{p, N S} \cdot\left(q_{d}=0\right) \\
G_{p, 1} \cdot\left(q_{d}>0\right)+G_{p, 2} \cdot\left(q_{d}<0\right)+G_{p, N S} \cdot\left(q_{d}=0\right)
\end{array}\right]
$$

Similarly to the roll rate controller, $G$ represents the bilinear interpolated value from an optimised gain set and the first subscript represents the corresponding controller component. However, the second subscript represents whether the gain surface is primary-positive, primary-negative or a neutral surface, $(1,2, N S)$.

\subsection{Normalised continuous multi-gain-surfaces}

Throughout the benchmark and CMGS approaches, the gain surfaces were obtained through optimisation of the maximum possible input for the given trim point flight conditions. As a result, if the reference signal demands a roll rate of $10 \mathrm{deg} / \mathrm{s}$, the same controller gains are implemented as for a roll demand capable of achieving up to $360 \mathrm{deg} / \mathrm{s}$, given the state dynamics. This increases the controller effort that is necessarily required to attain the demanded input, with the controller saturating beyond its capabilities. We now propose, therefore, that performing normalisation across the flight envelope for the maximum input demand against the gain set would allow for a dimensionless quantity to be established. This dimensionless quantity can then be scaled based on the input vector demand, and compared to the corresponding gain set that was obtained for the CMGS approach. The new methodology, normalised-continuous multi-gain-surface (NCMGS), is an adaptive approach that is a development of the previous three techniques. It still maintains bilinear interpolation between its primary and secondary gain sets and will, therefore, generate a continuous gain profile between neighbouring trim points, whilst also switching to a more appropriate gain surface based on the input vector (Figure 9).

One potential issue with NCMGS occurs during small inputs; inputs that could be pilot correctional manoeuvres with small magnitudes. This can result in the controller gains being so small as to not generate the desired dynamic response. We have overcome this problem by monitoring the input vector and the scaled bilinear interpolation of the primary gain set against a minimum value obtained from the neutral surface as derived through the CMGS approach. This provides the controller with a floor below which the gains cannot be reduced, allowing the normalised controller to maintain dynamic performance under small inputs. The logic equation for the NCMGS roll rate controller is 


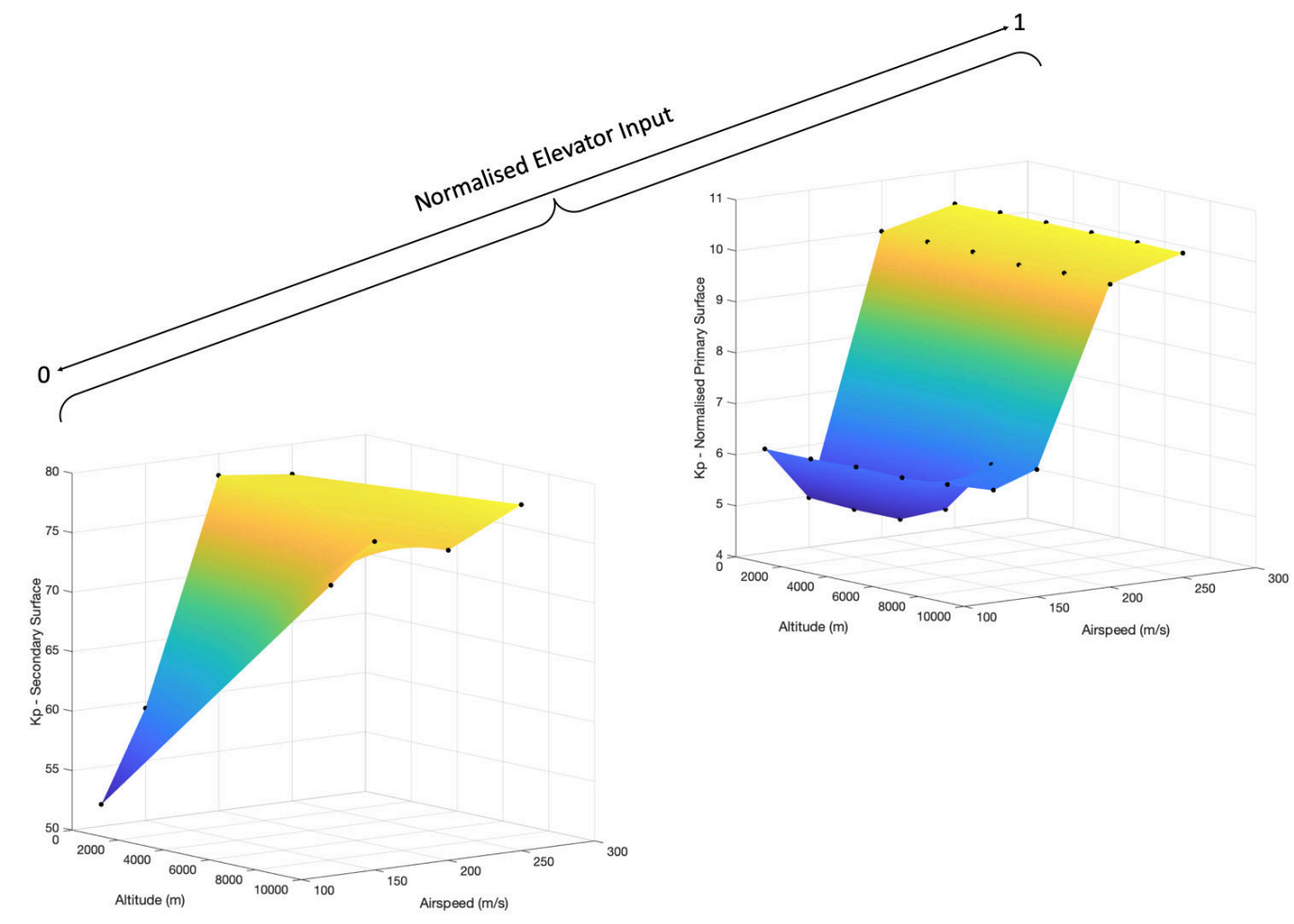

Figure 9: Continuous multi-gain surfaces implemented on the positive axis of the elevator. This shows the primary-positive and secondary gain surfaces acting as the bounds of the positive pitch rate hypersurface.

$$
\left[\begin{array}{c}
k_{p} \\
k_{i} \\
k_{d}
\end{array}\right]=\left[\begin{array}{c}
G_{p, 1} \cdot\left(\left|p_{d}\right| \neq 0 \wedge G_{p, N S}<G_{p, 1}\right)+G_{p, N S} \cdot\left(p_{d}=0 \vee G_{p, N S} \geq G_{p, 1}\right) \\
G_{i, 1} \cdot\left(\left|p_{d}\right| \neq 0 \wedge G_{i, N S}<G_{i, 1}\right)+G_{i, N S} \cdot\left(p_{d}=0 \vee G_{i, N S} \geq G_{i, 1}\right) \\
G_{d, 1} \cdot\left(\left|p_{d}\right| \neq 0 \wedge G_{d, N S}<G_{d, 1}\right)+G_{d, N S} \cdot\left(p_{d}=0 \vee G_{d, N S} \geq G_{d, 1}\right)
\end{array}\right] \times p_{d}
$$

where $G$ represents the normalised bilinear interpolated value from the optimised gain set. The first subscript to $G$ describes the corresponding controller component $(p, i, d)$, and the second subscript describes whether the gain set is primary (1) or the neutral surface (NS). For example, when attempting to determine the proportional gain from Equation 13, the primary gain surface $\left(G_{p, 1}\right)$ is activated when the magnitude of the roll rate demand $\left(\left|p_{d}\right|\right)$ does not equal zero, or if the corresponding gain for the primary surface is greater than the corresponding gain for the neutral surface $\left(G_{p, N S}<G_{p_{1}}\right)$. For the pitching moment, 


$$
\left[\begin{array}{c}
k_{p} \\
k_{i} \\
k_{d}
\end{array}\right]=\left[\begin{array}{c}
G_{p, 1} \cdot\left(q_{d}>0 \wedge G_{p, 3}<G_{p, 1} \wedge\left|q_{d}\right| \geq \Lambda\right)+G_{p, 2} \cdot\left(q_{d}<0 \wedge\left|q_{d}\right| \leq \Lambda\right)+G_{p, 3} \cdot\left(\left|q_{d}\right|<\Lambda \vee G_{p, 3}>G_{p, 1} \vee G_{p, 3}>G_{p, 2}\right) \\
G_{i, 1} \cdot\left(q_{d}>0 \wedge G_{i, 3}<G_{i, 1} \wedge\left|q_{d}\right| \geq \Lambda\right)+G_{i, 2} \cdot\left(q_{d}<0 \wedge\left|q_{d}\right| \leq \Lambda\right)+G_{i, 3} \cdot\left(\left|q_{d}\right|<\Lambda \vee G_{p, 3}>G_{p, 1} \vee G_{p, 3}>G_{p, 2}\right) \\
G_{d, 1} \cdot\left(q_{d}>0 \wedge G_{d, 3}<G_{d, 1} \wedge\left|q_{d}\right| \geq \Lambda\right)+G_{d, 2} \cdot\left(q_{d}<0 \wedge\left|q_{d}\right| \leq \Lambda\right)+G_{d, 3} \cdot\left(\left|q_{d}\right|<\Lambda \vee G_{p, 3}>G_{p, 1} \vee G_{p, 3}>G_{p, 2}\right)
\end{array}\right] \times q_{d}
$$

where the nomenclature is the same as for the roll rate controller. However, since three gain surfaces exist for the pitch rate controller the logic is not the same as the roll rate controller. Due to the larger moment of inertia and asymmetries about the longitudinal axis, a variable $\Lambda$ has been introduced. This variable represents the minimum pitch rate demand that can trigger a surface change. For example, when determining the proportional gains, the primary-positive gain surface $\left(G_{p, 1}\right)$ is activated if the reference signal $\left(q_{d}\right)$ is greater than zero and greater than $\Lambda$, and the corresponding primary-positive gain is greater than the neutral surface gain $\left(G_{p, N S}<G_{p, 1}\right)$. The primary-negative $\left(G_{p, 2}\right)$ surface is activated when the reference signal is less than zero and the magnitude of the reference signal is greater than $\Lambda$. Finally, the secondary, neutral gain, surface is activated if the magnitude of the reference signal is less than $\Lambda$ or if the corresponding gain for the neutral surface $\left(G_{p, N S}\right)$ is greater than the primary-positive or primary-negative surface gains. The resultant gain is then scaled by the reference signal $\left(q_{d}\right)$ due to the normalisation of the gain surfaces.

\section{Results}

In this section we apply CMGS and NCMGS to the aircraft model and perform the same simulation procedures that were described in Section 3. The response time for each of the manoeuvres as well as the steady-state error and controller efforts are evaluated.

The benchmark results, discussed in Appendix A, show that the implementation of CGS did not guarantee improvements over GS due to its dependence on the aircraft flight conditions. The dynamic performance was inconsistent and was highly dependant on which side of the trim point the aircraft was operating. By introducing a secondary gain surface, together with normalised gain scaling, we expect to eliminate this performance uncertainty and guarantee adequate operating conditions throughout the flight envelope.

The discussion of the results refers to the rise-time, fall-time and steady-state errors for the two benchmark approaches (GS and CGS) and the two stages of our novel approach (CMGS and NCMGS). The rise-time has been taken as the total time 
for the amplitude to change from $10 \%$ to $90 \%$ of steady-state conditions. The fall-time represents the removal of the step input and measures the controller's ability to return back to zero. Fall-time is calculated as the time taken for the amplitude to change from $90 \%$ to $10 \%$ steady-state conditions.

\subsection{Roll rate controller}

When analysing the benchmark results for the roll rate controller, based on the sample time history (Figure A.18) it is not apparent that a steady-state error is present due to the scaling of the $y$-axis. However, it can be seen in Table 2 that a steady-state error of between $0.24 \mathrm{deg} / \mathrm{s}$ and $0.50 \mathrm{deg} / \mathrm{s}$ is present for the response to the step input and between $0.03 \mathrm{deg} / \mathrm{s}$ and $0.14 \mathrm{deg} / \mathrm{s}$ during the return to zero demand following the step input. Whilst a steady-state error in the roll rate of the aircraft during a non-zero demand will have an impact on the time taken to achieve a specific roll attitude, it is significantly more important that the steady-state error upon return to a zero-rate state is small otherwise the aircraft will begin to slowly rotate.

\begin{tabular}{|c|c|c|c|c|c|}
\hline \multirow[b]{2}{*}{ Maneouvre } & \multirow[b]{2}{*}{ Method } & \multicolumn{2}{|c|}{ Rise Period } & \multicolumn{2}{|c|}{ Fall Period } \\
\hline & & $\begin{array}{l}\text { Rise time } \\
\text { (s) }\end{array}$ & $\begin{array}{l}\text { Steady-state } \\
\text { error }(\mathrm{deg} / \mathrm{s})\end{array}$ & $\begin{array}{l}\text { Rise time } \\
\text { (s) }\end{array}$ & $\begin{array}{l}\text { Steady-state } \\
\text { error }(\mathrm{deg} / \mathrm{s})\end{array}$ \\
\hline 1 & & 0.37 & 0.24 & 0.26 & 0.14 \\
\hline 2 & GS & 0.49 & 0.20 & 0.43 & 0.07 \\
\hline 3 & & 0.60 & 0.50 & 0.48 & 0.03 \\
\hline 1 & & 0.35 & 0.24 & 0.28 & 0.18 \\
\hline 2 & CGS & 0.46 & 0.20 & 0.43 & 0.12 \\
\hline 3 & & 0.57 & 0.30 & 0.45 & 0.12 \\
\hline 1 & & 0.35 & 0.24 & 0.11 & 0.01 \\
\hline 2 & CMGS & 0.46 & 0.20 & 0.21 & 0.01 \\
\hline 3 & & 0.57 & 0.30 & 0.24 & 0.02 \\
\hline 1 & & 0.16 & 0.10 & 0.11 & 0.01 \\
\hline 2 & NCMGS & 0.28 & 0.30 & 0.21 & 0.01 \\
\hline 3 & & 0.41 & 0.10 & 0.24 & 0.02 \\
\hline
\end{tabular}

Table 2: Roll rate manoeuvre results.

Figure 10 shows that during the initial step input the controller gains are identical for CGS and CMGS. This is because CMGS is only introducing a secondary surface when the step input is small or approaching zero. To analyse the results of CMGS, therefore, we focus solely on the fall time and steady-state error of the dynamic response when the step input is removed. 


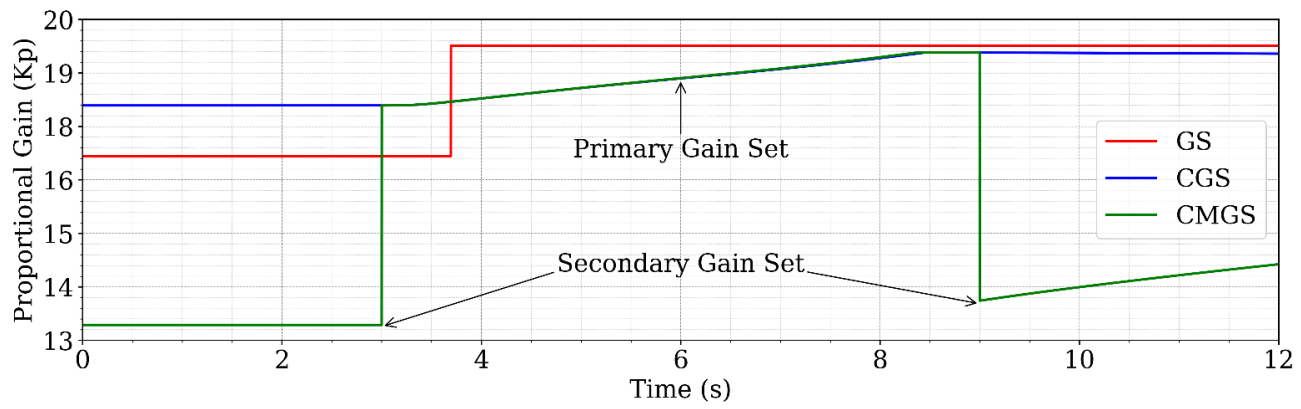

Figure 10: CMGS roll rate manoeuvre 1: proportional controller effort (the NCMGS response is not shown as it is identical to CMGS).

It can be seen in Figures 11(a)-11(c) that the steady-state error for GS and CGS appears to be growing over time. This steady-state error is largely due to the magnitude of the controller inputs not being suited to the given flight conditions.

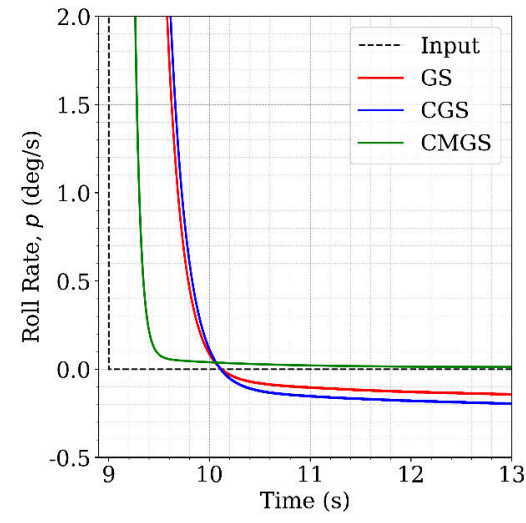

(a) Manoeuvre 1.

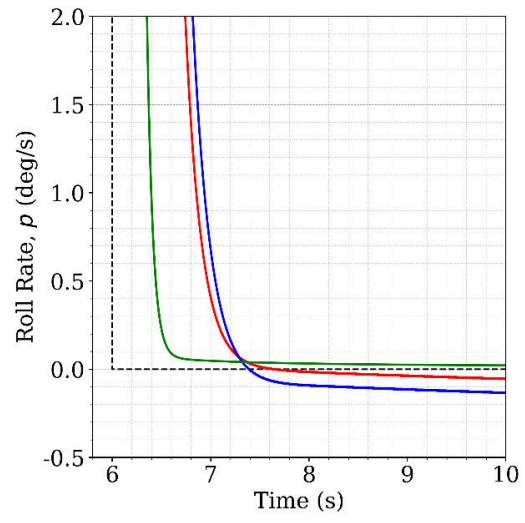

(b) Manoeuvre 2.

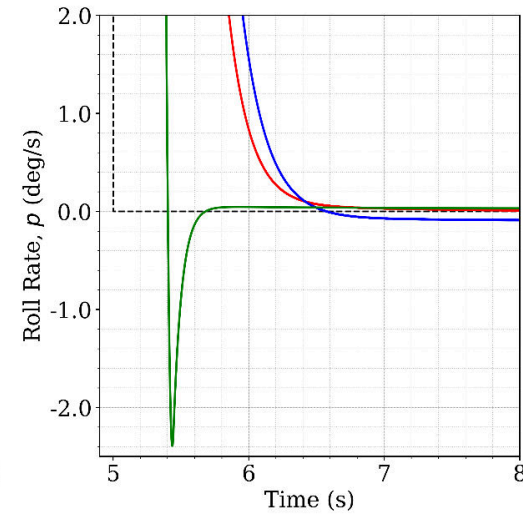

(c) Manoeuvre 3.

Figure 11: Roll rate manoeuvres; CMGS fall time and steady-state error analysis.

Not only has the CMGS controller effort been reduced during regions of low-to-zero input demand, the transient portion of the response has been reduced for all three manoeuvres and significant improvements to the steady-state error have been achieved (Figure 11). Table 2 shows that the fall-time for CMGS versus GS has been reduced on average by approximately $0.2 \mathrm{~s}$ across all three manoeuvres. The steady-state error has also been reduced on average by $71 \%$ for CMGS over GS. These improvements to the dynamics have been achieved whilst also reducing the controller effort.

Despite the improvements made by CMGS, we can see from Figure 11(c) that an overshoot has occurred during the transient stage. This overshoot was relatively small compared to the input, (less than $2 \%$ ) and did not induce any dynamic instability. The controller dynamics reduce the overshoot in less than $0.25 \mathrm{~s}$ and achieve steady-state with only $0.02 \mathrm{deg} / \mathrm{s}$ 
error.

Having proven the benefits of the CMGS gains for the roll rate controller during applications where the rate demand is low, we now focus on improving the dynamic response during the manoeuvre using NCMGS. As this approach normalises and then scales the controller gains based on the reference signal, we can further analyse these regions for improvements to the controller effort. It can be seen from Table 2 and Figure 12 that by implementing NCMGS we have been able to reduce the rise-time for all three manoeuvres. We see improvements of over $56 \%$ for manoeuvre 1, $43 \%$ for manoeuvre 2 and $33 \%$ for manoeuvre 3 compared to GS, CGS and CMGS. Furthermore, the steady-state error has been decreased for not only the rise period, but the fall period as well. The improvements made to the fall period and steady-state error through CMGS are still present with NCMGS. We do note, however, that the steady state error for the rise period of Manoeuvre 2 is higher for NCMGS than the other techniques and is discussed in Section 5.2. Nevertheless, this highlights the strength of scaling a normalised set of gains against the reference signal, as well as introducing logic to select a suitable gain surface.

Finally, when we analyse the controller effort (Figure 13) for the roll manoeuvre we see improvements again for NCMGS. The controller effort required to perform the manoeuvre successfully scales all three controller components and does not result in controller saturation occurring.

\subsection{Pitch Rate Controller}

As with the roll rate controller, we are not expecting to see improvements to the rise-time for GS and CGS during step input manoeuvres through the addition of CMGS. We are only expecting to see improvements to the fall-time once the step input has been removed. It can be seen in Figure 14 that the addition of the second surface has improved the fall-time during the removal of a step input, although when operating close to an optimised trim point, the differences are negligible. However, as the aircraft moves further away from an optimised trim point we see significant improvements.

Figure 14 and Table 3, show that through the addition of CMGS the fall-times for the removal of step responses have been reduced on average by $24 \%$ over GS and $26 \%$ over CGS (with a maximum improvement of $0.3 \mathrm{~s}$ for the second input). This reduction in the fall-time has been achieved despite a reduction of $37 \%$ in the proportional controller effort (Figure 15(a)), $10 \%$ in the integral controller effort, and $30 \%$ in the derivative controller effort (Figure 15(b)) when compared to GS. Similar reductions were seen when compared to CGS with a reduction of $32 \%$ in the proportional controller effort, $9 \%$ in the integral controller effort, and $32 \%$ in the derivative controller effort. These improvements to the dynamic response fall-time and the controller effort have all been achieved through changing the operating gain set based on the flight dynamics and reference signal. Having significantly reduced the fall-time and controller effort through the addition of a second gain surface using 


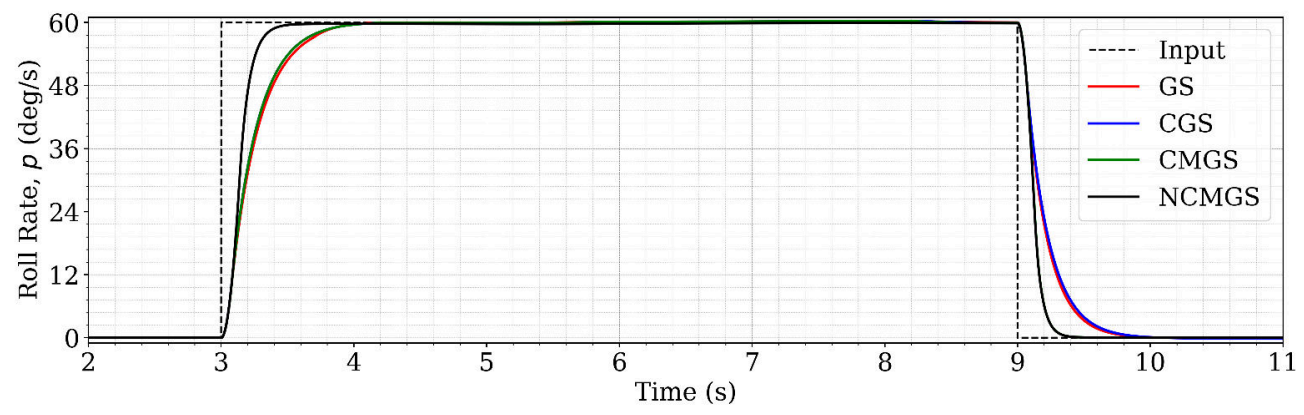

(a) Roll rate manoeuvre 1

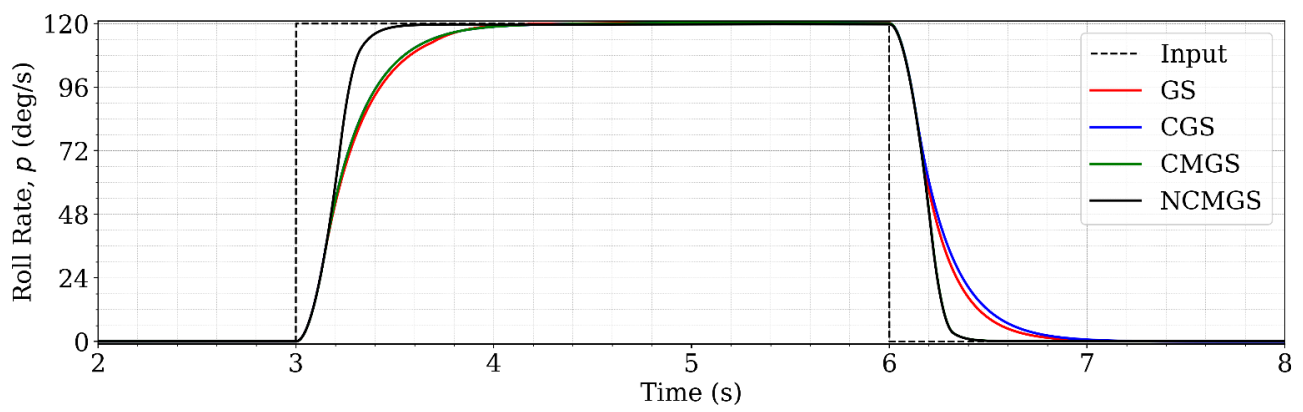

(b) Roll rate manoeuvre 2

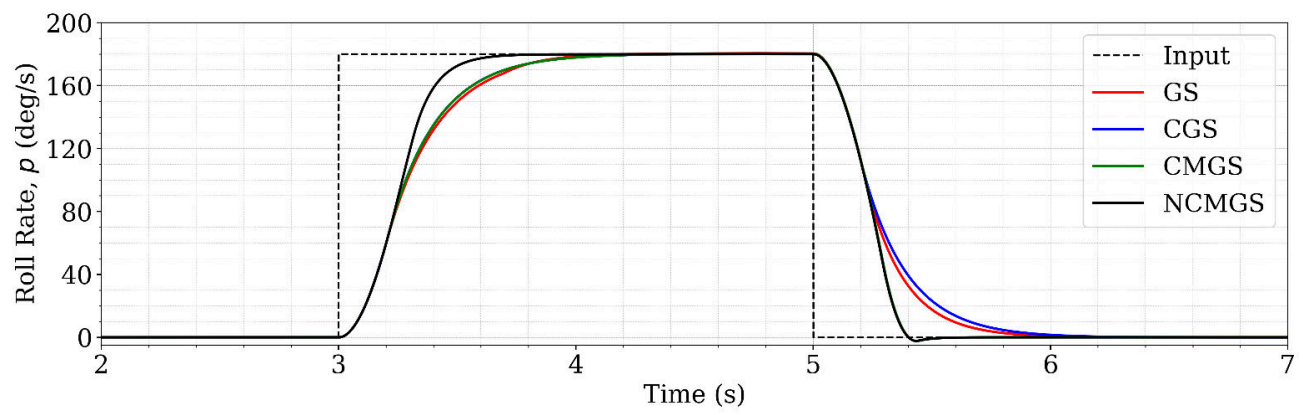

(c) Roll rate manoeuvre 3 .

Figure 12: Roll rate manoeuvres showing improvements in rise time, fall time and steady-state error for NCMGS.

CMGS, we reduced the rise-time of the step inputs and added further reductions to the controller effort through NCMGS.

It is apparent from Figure 16 that the rise-time of each of the step inputs has improved with NCMGS when compared to CMGS. The rise-time has been reduced by over $15 \%$ for the first step input, $34 \%$ for the second input, $38 \%$ for the third input, and $46 \%$ for the fourth step input. This demonstrates that NCMGS increases its effectiveness when moving further away from an optimised trim point. These reductions have been made despite also reducing the proportional controller effort by $31 \%$ (Figure 15(a)), the integral controller effort by $39 \%$, and the derivative controller effort by over $58 \%$ (Figure 15(b)) 


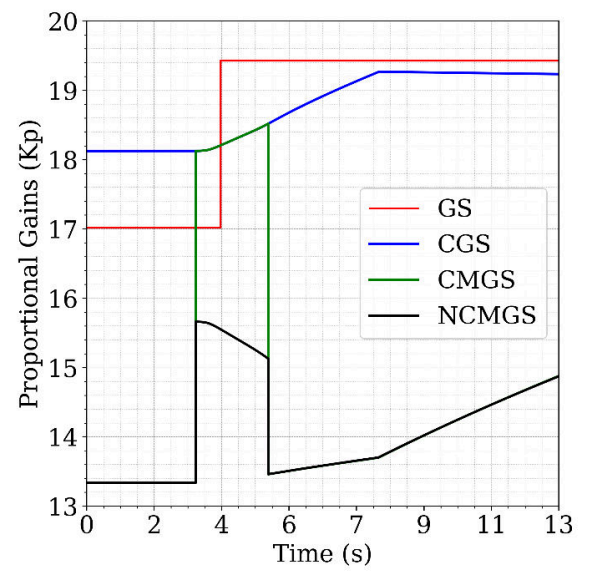

(a) Proportional controller effort.

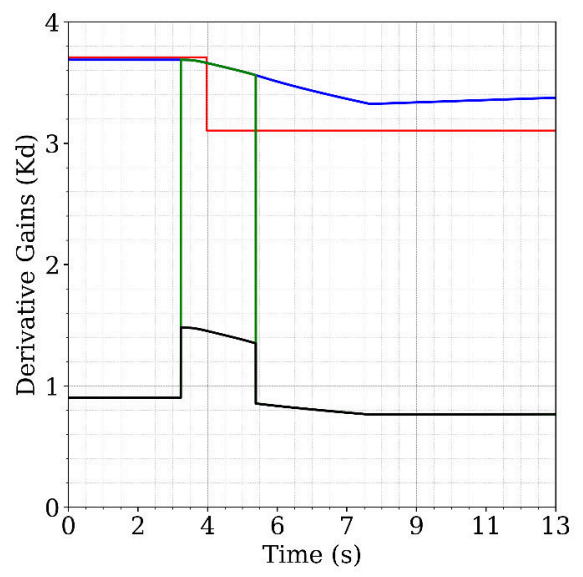

(b) Derivative controller effort.

Figure 13: Roll rate manoeuvre 3; $180 \mathrm{deg} / \mathrm{s}$ stepped input controller efforts.

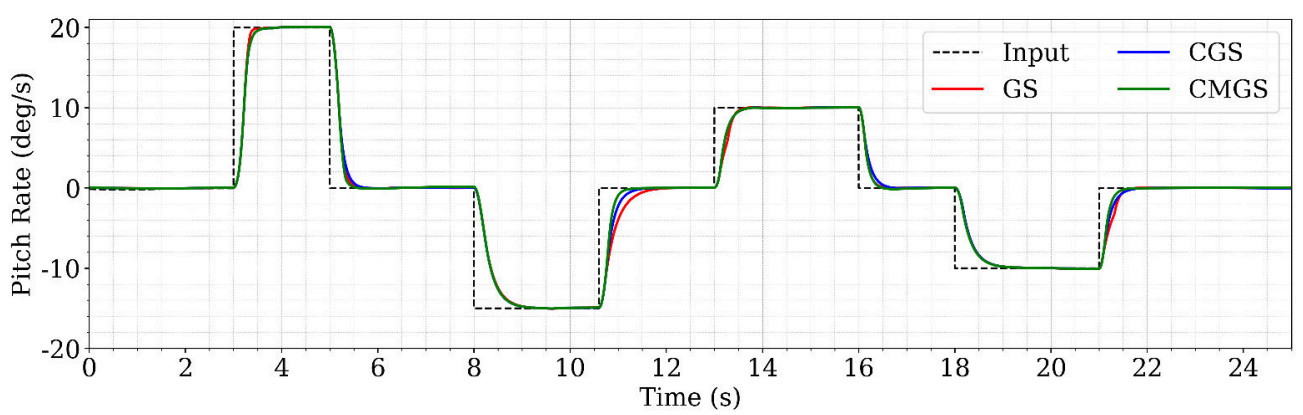

Figure 14: Pitch rate manoeuvre; CMGS dynamic response.

compared to CMGS.

Referring to Table 3, the steady-state error associated with CMGS and NCMGS has not necessarily shown improvements when compared to GS and CGS. Whilst it is expected that the integral component of the PID controller would eliminate the steady-state error of the system, it is not able to compensate fully for the non-linearity in the aircraft aerodynamics. The integral gain in the pitch rate controller will be sufficient at some attitudes for the aircraft to converge on the demanded angular rate quickly. At other attitudes the integral gain may not be sufficient for this to occur. This affects the steady-state error over time.

A potential solution to this problem is to add a fourth dimension to the gain surfaces so that the controller can be scheduled with either pitch angle or angle of attack, as well as the current scheduling against altitude and airspeed. It was concluded by Yang et al. [5] that a controller scheduled solely against pitch rate does not produce adequate performance as 


\begin{tabular}{|c|c|c|c|c|c|}
\hline \multirow[b]{2}{*}{ Step input } & \multirow[b]{2}{*}{ Method } & \multicolumn{2}{|c|}{ Rise Period } & \multicolumn{2}{|c|}{ Fall Period } \\
\hline & & $\begin{array}{l}\text { Rise time } \\
\text { (s) }\end{array}$ & $\begin{array}{l}\text { Steady-state } \\
\text { error (deg/s) }\end{array}$ & $\begin{array}{l}\text { Rise time } \\
\text { (s) }\end{array}$ & $\begin{array}{l}\text { Steady-state } \\
\text { error }(\mathrm{deg} / \mathrm{s})\end{array}$ \\
\hline $1(+20 \mathrm{deg} / \mathrm{s})$ & \multirow{4}{*}{ GS } & 0.21 & 0.02 & 0.24 & 0.02 \\
\hline $2(-15 \mathrm{deg} / \mathrm{s})$ & & 0.52 & 0.02 & 0.57 & 0.03 \\
\hline $3(+10 \mathrm{deg} / \mathrm{s})$ & & 0.33 & 0.02 & 0.24 & 0.02 \\
\hline $4(-10 \mathrm{deg} / \mathrm{s})$ & & 0.60 & 0.02 & 0.38 & 0.04 \\
\hline $1(+20 \mathrm{deg} / \mathrm{s})$ & \multirow{4}{*}{ CGS } & 0.24 & 0.05 & 0.29 & 0.04 \\
\hline $2(-15 \mathrm{deg} / \mathrm{s})$ & & 0.50 & 0.05 & 0.37 & 0.01 \\
\hline $3(+10 \mathrm{deg} / \mathrm{s})$ & & 0.33 & 0.04 & 0.31 & 0.01 \\
\hline $4(-10 \mathrm{deg} / \mathrm{s})$ & & 0.51 & 0.02 & 0.36 & 0.02 \\
\hline $1(+20 \mathrm{deg} / \mathrm{s})$ & \multirow{4}{*}{ CMGS } & 0.24 & 0.05 & 0.22 & 0.05 \\
\hline $2(-15 \mathrm{deg} / \mathrm{s})$ & & 0.49 & 0.05 & 0.27 & 0.01 \\
\hline $3(+10 \mathrm{deg} / \mathrm{s})$ & & 0.32 & 0.02 & 0.24 & 0.07 \\
\hline $4(-10 \mathrm{deg} / \mathrm{s})$ & & 0.50 & 0.01 & 0.25 & 0.02 \\
\hline $1(+20 \mathrm{deg} / \mathrm{s})$ & \multirow{4}{*}{ NMCGS } & 0.21 & 0.04 & 0.22 & 0.03 \\
\hline $2(-15 \mathrm{deg} / \mathrm{s})$ & & 0.33 & 0.01 & 0.27 & 0.02 \\
\hline $3(+10 \mathrm{deg} / \mathrm{s})$ & & 0.20 & 0.04 & 0.23 & 0.08 \\
\hline $4(-10 \mathrm{deg} / \mathrm{s})$ & & 0.27 & 0.05 & 0.26 & 0.01 \\
\hline
\end{tabular}

Table 3: Pitch rate manoeuvre results.

it cannot compensate for aerodynamic coupling. Whilst the addition of a pitch angle scheduler could have some benefits for reducing the steady-state error, scheduling against quickly-varying dynamic states can result in the dynamics transitioning too quickly between trim points inducing instabilities $[4,6]$. Given that the controller being investigated is an angular rate controller with a steady-state error of less than $0.1 \mathrm{deg} \cdot \mathrm{s}^{-1}$, there is little benefit in implementing a fourth dimension to the gain scheduler. Furthermore, since the angular rate controllers have been designed to be operated by a human pilot (who can manually adjust the controls to compensate), or an autonomous vehicle (which will have an attitude autopilot implemented outside of the rate loop) this steady-state error is not overwhelmingly detrimental to the aircraft's performance.

\section{Conclusions}

A series of benchmark step input responses for gain scheduling (GS) and continuous gain scheduling (CGS) methodologies were obtained for a six degree of freedom non-linear aircraft model. Through novel adaptations of the gain selection criteria, namely continuous multi-gain surfaces (CMGS) and normalised continuous multi-gain surfaces (NCMGS) we have been able to not only improve the controller dynamics, but also achieve significant reductions in the controller effort required to 


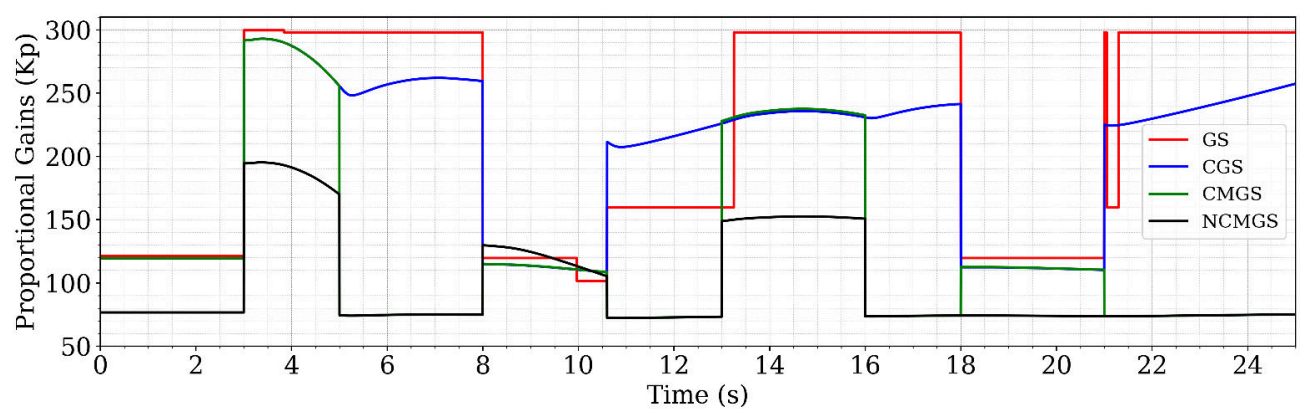

(a) Proportional controller effort.

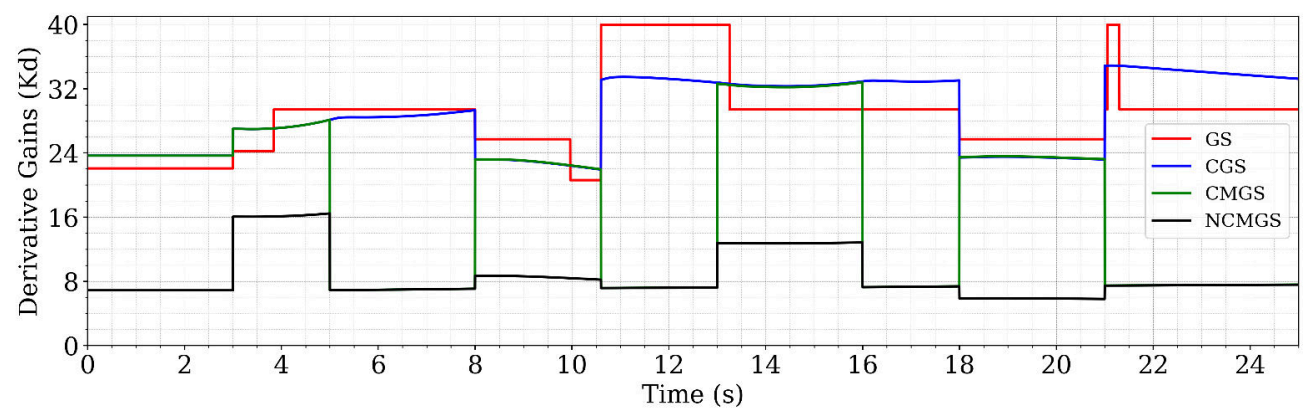

(b) Derivative controller effort.

Figure 15: Controller effort for the pitch rate manoeuvre.

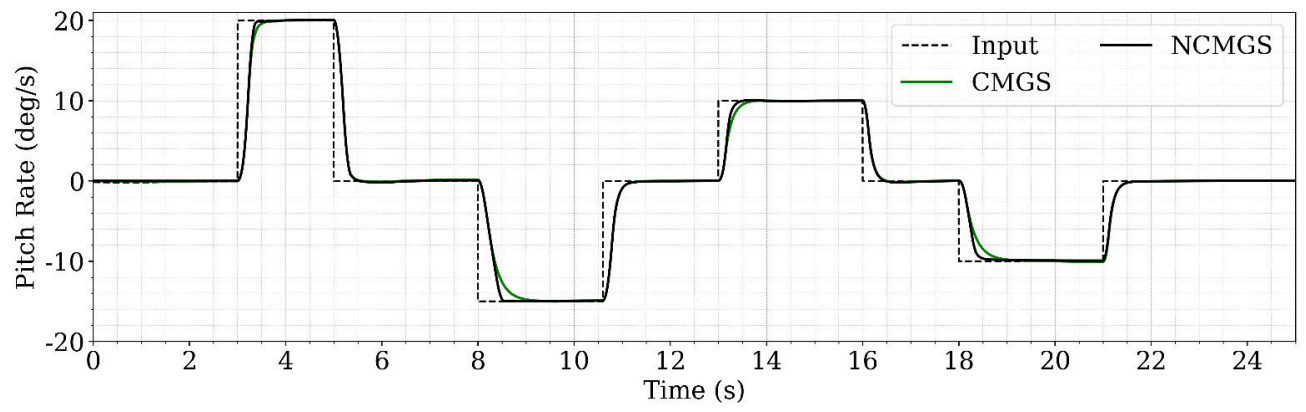

Figure 16: Pitch rate manoeuvre; NCMGS dynamic response compared to CMGS.

perform all of the manoeuvres considered.

It was found that GS and CGS were inconsistent in terms of controller performance. The architecture is dependant solely on the aircraft flight conditions whereby any dynamic state changes throughout the manoeuvre can have overwhelming implications on the output. We have alleviated this in the pitch controller by adding a second layer of gains to not only reduce the fall-time of all step inputs but also provide significant reductions to the corresponding controller effort. Furthermore, we 
have improved the rise-time for all step inputs throughout the pitch rate manoeuvres, regardless of the given aircraft flight conditions, through the addition of NCMGS.

The gain normalisation and gain-scaling functions applied through NCMGS have successfully improved the rise time for low, medium and high input demand manoeuvres to the roll rate controller. Furthermore, the second layer of gains implemented through CMGS has provided significant improvements to the fall-time upon the removal of the stick input. This improvement allows the aircraft to be operated in a more aggressive but controllable manner, as the controller is able to respond up to $50 \%$ faster in reducing the angular velocity of the aircraft.

\section{References}

[1] W. J. Rugh and J. S. Shamma. Research on gain scheduling. Automatica, 36(10):1401-1425, 2000. doi: 10.1016/ S0005-1098(00)00058-3.

[2] J. S. Shamma and M. Athans. Guaranteed properties of gain scheduled control for linear parameter-varying plants. Automatica, 27(3):559-564, 1991. doi: 10.1016/0005-1098(91)90116-J.

[3] R. T. Reichert. Dynamic scheduling of modern-robust-control autopilot designs for missiles. IEEE Contr. Syst. Mag., 12 (5):35-42, Oct 1992. doi: 10.1109/37.158896.

[4] T. Richardson, P. Davison, M. Lowenberg, and M. di Bernardo. Control of nonlinear aircraft models using dynamic state-feedback gain scheduling. In AIAA Guidance, Navigation, and Control Conference and Exhibit, number AIAA 2003-5503, Austin, TX, 11-14 August 2003. doi: 10.2514/6.2003-5503.

[5] W. Yang, M. N. Hammoudi, G. Herrmann, M. Lowenberg, and X. Chen. Two-state dynamic gain scheduling control applied to an F16 aircraft model. Int. J. Nonlin. Mech., 47(10):1116-1123, 2012. doi: 10.1016/j.ijnonlinmec.2011.09.007.

[6] R. A. Nichols, R. T. Reichert, and W. J. Rugh. Gain scheduling for h-infinity controllers: A flight control example. IEEE T. Contr. Syst. T., 1(2):69-79, 1993. doi: 10.1109/87.238400.

[7] M. W. McConley, B. D. Appleby, M. A. Dahleh, and E. Feron. A computationally efficient Lyapunov-based scheduling procedure for control of nonlinear systems with stability guarantees. IEEE T. Automat. Contr., 45(1):33-49, 2003. ISSN 00189286. doi: $10.1109 / 9.827354$. 
[8] B. Lu, F. Wu, and S. Kim. Switching LPV control of an F-16 aircraft via controller state reset. IEEE T. Contr. Syst. T., 14 (2):267-277, 2006. doi: 10.1109/tcst.2005.863656.

[9] A. T. Tran, N. Sakamoto, and K. Mori. Nonlinear gain-scheduled flight controller design via stable manifold method. Aerosp. Sci. Technol., 80:301-308, 2018. doi: 10.1016/j.ast.2018.07.002.

[10] D. J. Stilwell and W. J. Rugh. Interpolation of observer state feedback controllers for gain scheduling. IEEE T. Automat. Contr., 44(6):1225-1229, 1999. doi: 10.1109/9.769379.

[11] D. Galvão Wall. A graph-theory-based C-space path planner for mobile robotic manipulators in close-proximitiy environments. PhD thesis, Cranfield University, Shrivenham, UK, 2016.

[12] S. Theodoulis, F. Sève, and P. Wernert. Robust gain-scheduled autopilot design for spin-stabilized projectiles with a course-correction fuze. Aerosp. Sci. Technol., 42:477-489, 2015. doi: 10.1016/j.ast.2014.12.027.

[13] Y. Choi, H. Jimenez, and D. N. Mavris. Statistical gain-scheduling method for aircraft flight simulation. Aerosp. Sci. Technol., 46:493-505, 2015. doi: 10.1016/j.ast.2015.08.011.

[14] F. Wu and S. Kim. LPV controller interpolation for improved gain-scheduling control performance. In AIAA Guidance, Navigation, and Control Conference and Exhibit, number 2002-4759, Monterey, CA, 5-8 August 2002. doi: 10.2514/6. 2002-4759.

[15] P. Apkarian and R. J. Adams. Advanced gain-scheduling techniques for uncertain systems. IEEE T. Contr. Syst. T., 6(1): 21-32, 1998.

[16] T. Shirnomura. Hybrid control of gain-scheduling and switching: A design example of aircraft control. In Proceedings of the 2003 American Control Conference, pages 4639-4644, Denver, CO, 4-6 June 2003. doi: 10.1109/ACC.2003.1242455.

[17] B. L. Stevens, F. L. Lewis, and E. N. Johnson. Aircraft Control and Simulation: Dynamics, Controls Design, and Autonomous Systems. John Wiley \& Sons Inc., Hoboken, NJ, USA, 2015. ISBN 9781119174882.

[18] Wikimedia Commons. F16 aircraft image, 2004. URL https://commons.wikimedia.org/wiki/File:F16_drawing. svg.

[19] L. T. Nguyen, M. E. Ogburn, W. P. Gilbert, K. S. Kibler, P. W. Brown, and P. L. Deal. Simulator study of stall/post-stall characteristics of a fighter airplane with relaxed longitudinal static stability. Technical report 1538, NASA, 1979. 
[20] B. Lu. Linear Parameter-Varying Control of an F-16 Aircraft at High Angle of Attack. PhD thesis, North Carolina State University, 2004.

[21] A. Savran, R. Tasaltin, and Y. Becerikli. Intelligent adaptive nonlinear flight control for a high performance aircraft with neural networks. ISA T., 45(2):225-247, 2006. doi: 10.1016/S0019-0578(07)60192-X.

[22] A. Omran, B. Newman, and D. Landman. Global aircraft aero-propulsive linear parameter-varying model using design of experiments. Aerosp. Sci. Technol., 22(1):31-44, 2012. doi: 10.1016/j.ast.2011.05.008.

[23] E. A. Morelli. Global nonlinear parametric modeling with application to F-16 aerodynamics. In Proceedings of the 1998 American Control Conference, volume 2, pages 997-1001, Philadelphia, PA, 26 June 1998. doi: 10.1109/ACC.1998.703559.

[24] O. Albostan and M. Göka. Mode decoupling robust eigenstructure assignment applied to the lateral-directional dynamics of the F-16 aircraft. Aerosp. Sci. Technol., 77:677-687, 2018. doi: 10.1016/j.ast.2018.04.011.

[25] X. S. Yang. A new metaheuristic bat-inspired algorithm. In J. R. González, D. A. Pelta, C. Cruz, G. Terrazas, and N. Krasnogor, editors, Studies in Computational Intelligence, volume 284, pages 65-74. Springer, Berlin, Heidelberg, 2010. doi: 10.1007/978-3-642-12538-6_6.

\section{Appendix A. Benchmark results}

Having discussed GS, and also the application of generating a continuous gain profile through bilinear interpolation throughout Section 1, we have applied both methodologies to the aircraft model to develop a series of benchmark results. The dynamic states were scheduled against airspeed $(u)$ and altitude $(h)$. Both the GS and CGS techniques use the same gain sets obtained through optimisation, with CGS performing bilinear interpolation to generate a continuous gain-profile over the course of the manoeuvre. The following two subsections present the benchmark results, one for the roll rate controller and one for the pitch rate controller.

\section{Appendix A.1. Roll rate controller}

When analysing the benchmark results for the roll rate controller, we focussed on the rise-time for the step input, fall-time as the input is removed, and the steady-state errors throughout the manoeuvre. We will also briefly discuss the controller effort however, due to the optimisation approach, the controller effort is identical for all three benchmark manoeuvres. This 
is because the controller gains are scheduled against altitude and airspeed, and have no association with the magnitude of the manoeuvre. Hence, we only need to analyse the controller effort once for all three roll rate manoeuvres.

By inspecting Figure A.17 we can infer that during the application of the step input, the aircraft is not operating at a trim point. It is clear from the change in gain values for GS, that the aircraft transitions across two trim points, and the interpolation between them for CGS shows a gradient between two plateaus (in the proportional gain). However, the CGS gain values at these plateaus do not match those selected by the GS approach. It is clear, therefore, that the CGS interpolation is not using the exact trim points as inputs. This indicates that during the application of the step input the flight conditions for the aircraft are offset from the local trim point. As a result, interpolation occurs between two trim points causing the controller equipped with CGS gains to start with a slightly higher proportional gain and a slightly lower derivative gain than the GS controller. The gain configuration during this region of operation enables the controller equipped with CGS gains to achieve a $0.025 \mathrm{~s}$ reduction in rise-time, and a marginally reduced steady-state error (Figure A.18).

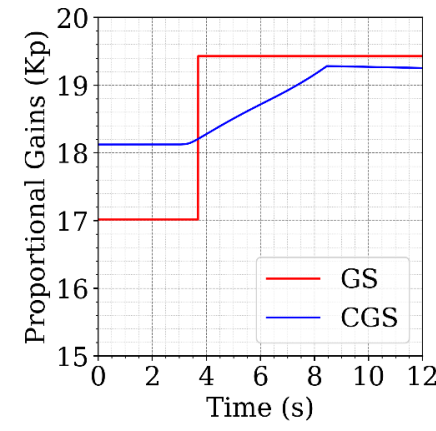

(a) Proportional controller gain.

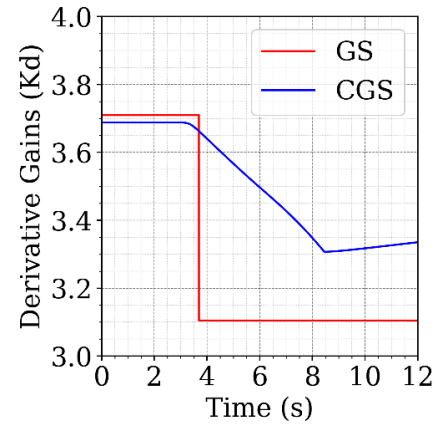

(b) Derivative controller gain.

Figure A.17: Proportional and derivative controller gains scheduled for the roll rate manoeuvre.

At approximately $3.7 \mathrm{~s}$ into the simulation, as the aircraft flight conditions move between two local trim points, a large instantaneous change to the GS controller gains is observed. This causes a decrease in the derivative gain and an increase in the proportional and integral components. As a result, when the step input is removed, the fall-time for CGS is on average $0.11 \mathrm{~s}$ slower than GS (Figure A.18). This illustrates a discrepancy within the gain architecture whereby the controller response is often a result of the current flight condition and hence CGS cannot guarantee an improvement to the controller performance across the flight envelope. We aim to reduce this discrepancy through our novel approach, which aims to guarantee an improvement to the roll rate throughout the flight envelope. 


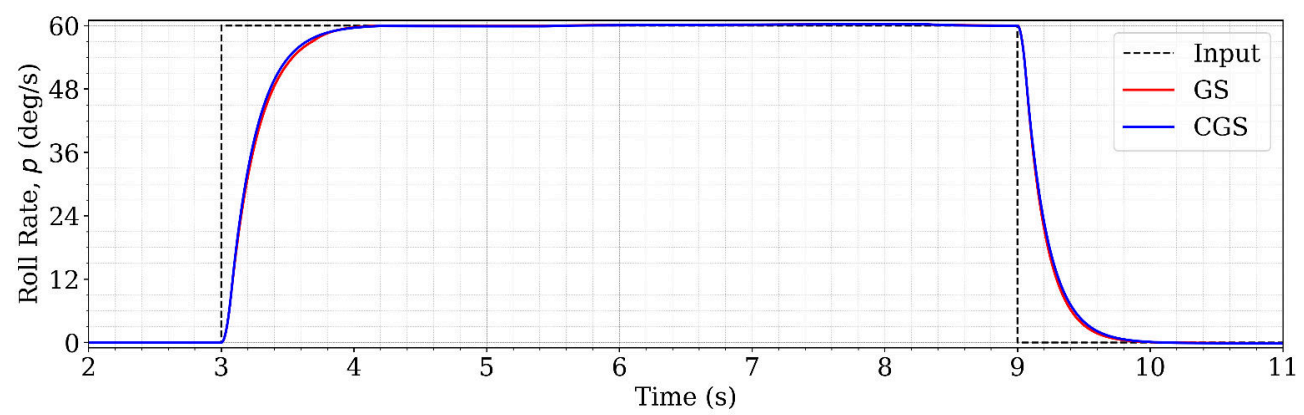

(a) Roll rate manoeuvre 1 .

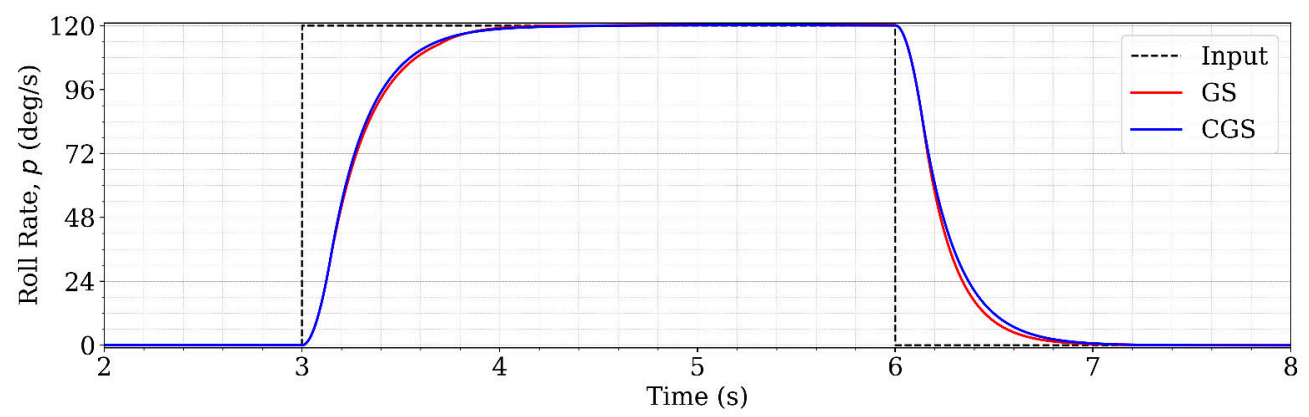

(b) Roll rate manoeuvre 2 .

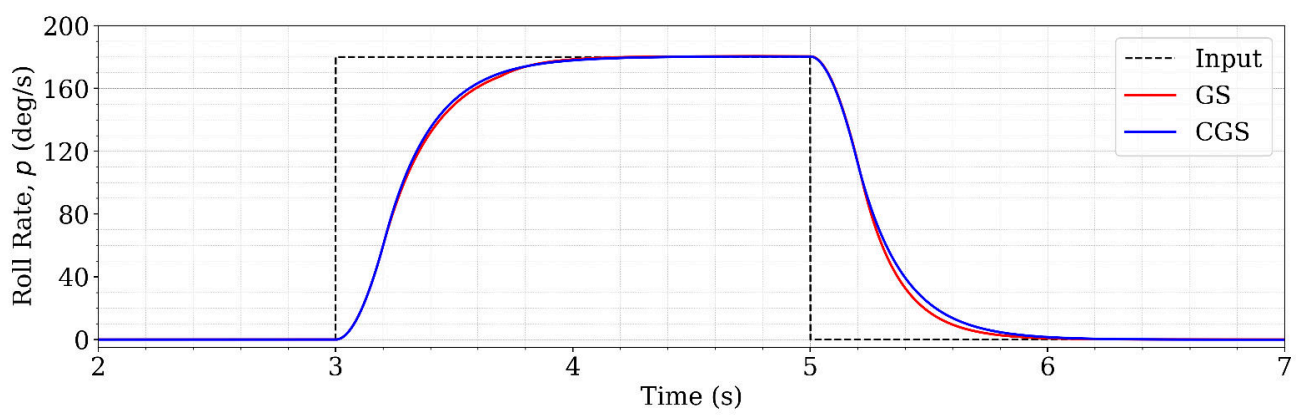

(c) Roll rate manoeuvre 3 .

Figure A.18: Roll rate response for GS and CGS benchmark results.

\section{Appendix A.2. Pitch rate controller}

Figure A.19 shows that during the pitch rate manoeuvre the aircraft is passing through multiple optimised trim points. Since the pitch rate controller is equipped with two gain sets, one for positive pitch rates and the other for negative pitch rates, the process of switching between the positive and negative pitch rate gain surfaces should not be confused with a change of gain sets across trim points. In a similar way to the roll rate controller, the aircraft flight conditions in relation to the local trim points during the manoeuvre are the determining factor for the dynamic response of the aircraft. Once 
again it can be inferred that during the first $3 \mathrm{~s}$ of the manoeuvre the aircraft is operating relatively close to a local trim point. During the remainder of the manoeuvre the divergence of the aircraft flight conditions from the optimised trim points results in significantly different proportional gain values between GS and CGS. Although not shown this is also true for the integral and derivative gains for the same controller. As the aircraft flight conditions move away from the local trim point (e.g. between $10.5 \mathrm{~s}$ and $13 \mathrm{~s}$ ) and bilinear interpolation increases the gains, we see a faster rise time from CGS (Figure A.20). However, in areas where CGS interpolates to a smaller gain magnitude (e.g. $16 \mathrm{~s}$ to $18 \mathrm{~s}$ ) the rise time for CGS is slower than for GS. This is the same effect that was experienced with the roll rate controller, and is a fundamental flaw of the GS and CGS architecture.

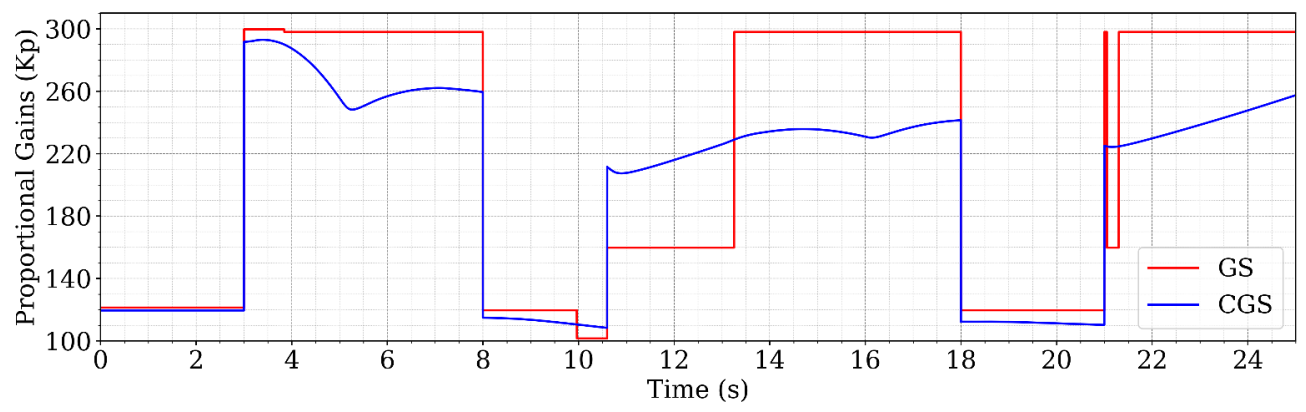

Figure A.19: Variation in proportional gain $\left(K_{p}\right)$ during the benchmark pitch manoeuvre.

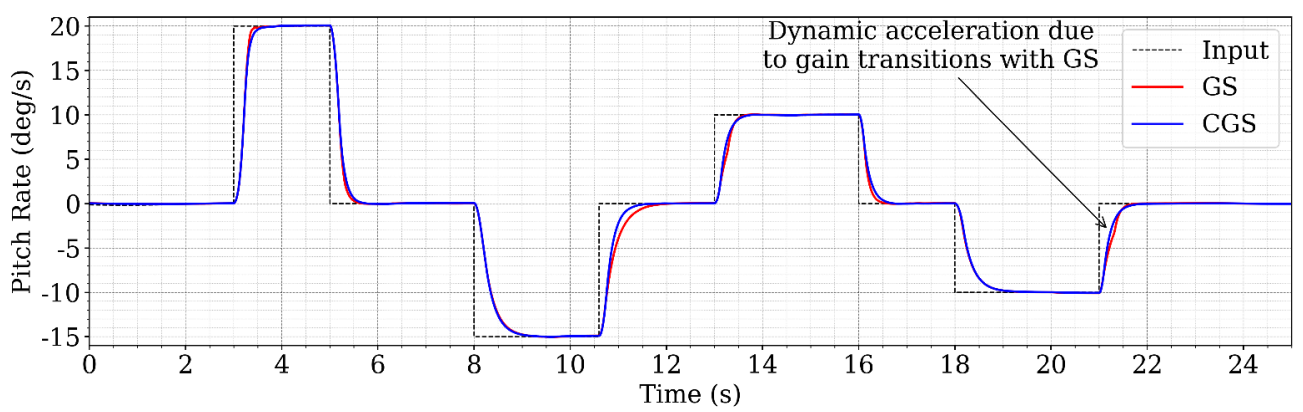

Figure A.20: System response using GS and CGS during the benchmark pitch manoeuvre.

When we analyse the proportional controller effort for the pitch rate manoeuvre (Figure A.19), we observe that, during the final step input at approximately $21 \mathrm{~s}$ into the simulation, the aircraft passes back and forth twice through a gain intersection. As shown in Figure A.20, this induces a rapid change to the controller and causes an acceleration to occur during the manoeuvre. Although this did not induce dynamic instability it is concerning that instantaneous gain switches can induce 
an acceleration, which under the right circumstances could cause instability.

When considering the airspeed over the entire manoeuvre, we can see from Figure 21(a) that until approximately $11 \mathrm{~s}$ both simulations have almost identical airspeeds. Beyond this point Figure A.19 shows that between $11 \mathrm{~s}$ and $14 \mathrm{~s}$ the aircraft is operating at flight conditions that fall between two trim points, resulting in an increased fall-time for the controller using GS (Figure A.20). This slow fall-time when compared to CGS results in the aircraft continuing to pitch down for an additional second. This reduces the overall pitch angle for the aircraft using GS, and induces a pitch angle offset from CGS, as seen in Figure 21(b). As the aircraft using GS is now performing the remaining manoeuvres at a slightly lower pitch angle, it loses less airspeed over the remainder of the simulation resulting in an ability to generate larger aerodynamic forces hence allowing a faster response.

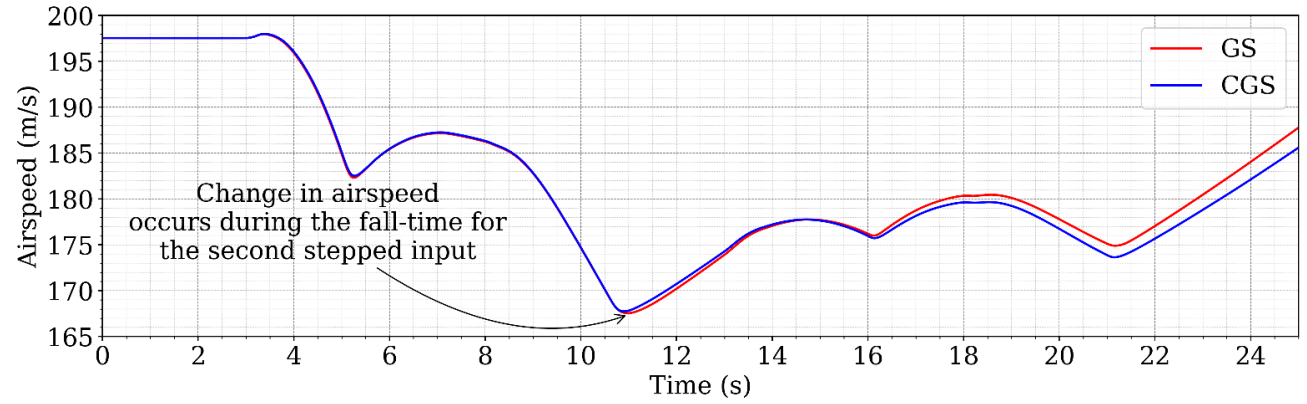

(a) Airspeed during the benchmark pitch rate manoeuvre.

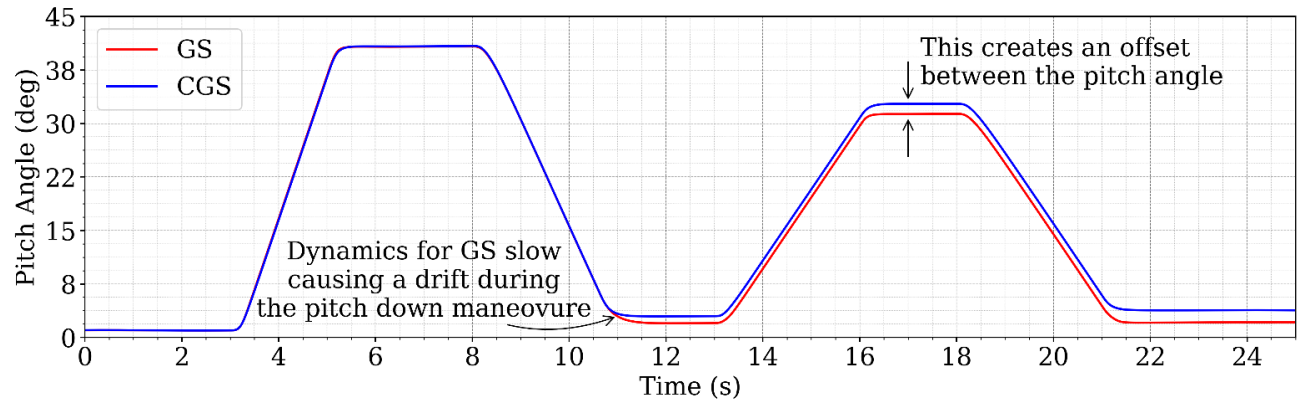

(b) Pitch angle during the benchmark pitch rate manoeuvre.

Figure A.21: Pitch rate manoeuvre dynamics for benchmark GS and CGS gains.

Having analysed the pitch rate manoeuvre dynamics for GS and CGS, it is difficult to draw any conclusions as to which methodology has a clear advantage. It is apparent that during the initial $11 \mathrm{~s}$, whilst operating close to a local trim point, the controller using GS has a slight advantage with regard to rise and fall times. This advantage is negligible and does not result in any noticeable differences between the airspeed and pitch angles of the two aircraft during this period. However, as the 
simulation progresses, and the flight conditions move away from the optimised trim point, the rise time increases for the GS controller. This causes the aircraft to react more slowly when compared to CGS. As a consequence, when the stick input is removed the controller takes longer to bring the aircraft dynamics back to zero degrees-per-second pitch rate, resulting in a longer pitch down motion at approximately $11 \mathrm{~s}$. This additional $0.5 \mathrm{~s}$ of pitch down motion induces an angular offset between both corresponding sets of aircraft dynamics in terms of the pitch angle, as seen in Figure 21(b). This angular offset means the controller using GS gains is now operating with a reduced pitch angle, decreasing aerodynamic drag. Since both controllers have the same throttle setting, the reduced drag for GS means the aircraft model is able to accelerate more quickly than CGS, increasing the airspeed. This can be seen in Figure 21(a); with the pitch angle offset clearly visible in Figure 21(b). 